Gazi University
Journal of Science
http://dergipark.gov.tr/gujs

\title{
Lower Separation Axioms in Čech Fuzzy Soft Closure Spaces
}

\author{
Rasha MAJEED $^{1, *}$ (iD) , Lina MAIBED ${ }^{1}$ (D) \\ ${ }^{1}$ University of Baghdad, Faculty of Education for Pure Sciences Abn Al-Haitham, Department of Mathematics,Baghdad, Iraq
}

\begin{abstract}
Highlights
- In this work, some properties of Cech fuzzy soft closure spaces are introduced and studied.

- Also, several types of lower separation axioms in Cech fuzzy soft closure space are introduced.

- In addition, the relationship between these types of separation axioms are discussed.

- Moreover, several examples are given to support our study.
\end{abstract}

\begin{tabular}{l} 
Article Info \\
\hline \\
Received: $12 / 06 / 2018$ \\
Accepted: $11 / 04 / 2019$ \\
Keywords \\
\hline Fuzzy soft set \\
Fuzzy soft point \\
Čech fuzzy soft operator \\
Distinct fuzzy soft points \\
$T_{i}$-Č ech closure spaces
\end{tabular}

\begin{abstract}
By considering $\check{C}$ ech fuzzy soft closure spaces $(X, \theta, K)$, we provide a basic structure of a fuzzy soft topological space $\left(X, \tau_{\theta}, K\right)$ associated with $\check{C}$ ech fuzzy soft closure space $(X, \theta, K)$. Separation axioms, namely, $T_{i}(i=0,1,2)$, semi- (respectively, pseudo and Uryshon) $T_{2}$ are studied in both $\check{C}$ ech fuzzy soft closure spaces and its associative fuzzy soft topological spaces. It is shown that hereditary property is satisfied for $T_{i}, i=0,1$ with respect to $\breve{C}$ ech fuzzy soft closure space but for other mentioned types of separations axioms, hereditary property satisfies for closed subspaces of $\breve{C}$ ech fuzzy soft closure space. Several examples are given to illustrate each type of the separation axioms and to study the relationship between them.
\end{abstract}

\section{INTRODUCTION}

The notion of fuzzy sets was firstly proposed by Zadeh [1] in 1965 as one of the effective mathematical tools to deal with uncertainties, where each element in a fuzzy set has a grade of membership. In 1999, Molodsov [2] initiated a new theory called soft set theory, by using classical sets, that also deals with uncertainties and ambiguity. A combination of fuzzy sets and soft sets, namely fuzzy soft sets, is then formulated by Maji et al. [3]. By using fuzzy soft sets, Tanay and Kandemir [4] defined fuzzy soft topology.

The concept of $\check{C}$ ech closure spaces $(X, \mathcal{C})$ was introduced by $\check{C}$ ech [5] in 1966, where $\mathcal{C}: P(X) \rightarrow P(X)$ is a mapping satisfying $\mathcal{C}(\varnothing)=\varnothing, A \subseteq \mathcal{C}(A)$ and $\mathcal{C}(A \cup B)=\mathcal{C}(A) \cup \mathcal{C}(B)$. The mapping $\mathcal{C}$ is referred to as $\check{C}$ ech closure operator on $X$. In 1985, Mashhour and Ghanim [6] introduced the concept of $\check{C}$ ech fuzzy closure space by replacing ordinary sets with fuzzy sets in the definition of $\check{C}$ ech closure space. On the other hand, in 2014, Gowri and Jegadeesan [7] and Krishnaveni and Sekar [8], have introduced and studied soft $\breve{C}$ ech closure spaces. Note that the soft closure operator in the sense of Gowri and Jegadeesan is defined from the power set $P\left(X_{F_{A}}\right)$ of $X_{F_{A}}$ to itself (where $F_{A}$ is a soft set over the universe set $X$ with the set of parameters $K$, and $A \subseteq K$ ) while, Krishnaveni and Sekar, defined soft closure operator from the set of all soft sets over $X$ to itself. Very recently, Majeed [9] generalized soft $\breve{C}$ ech closure space, in the sence of Krishnaveni and Sekar, into $\check{C}$ ech fuzzy soft closure spaces. Also, Majeed and Maibed [10] have further studied some structures of $\check{C}$ ech fuzzy soft closure spaces. Majeed and Maibed show that every $\check{C}$ ech fuzzy soft closure space gives a parameterized family of $\check{C}$ ech fuzzy closure spaces.

In the present paper, we continue studying the properties of $\check{C}$ ech fuzzy soft closure spaces and their separation axioms. Some of the provided properties of $\check{C}$ ech fuzzy soft closure spaces are essential for 
studying the separation axioms. In Section 3, basic structure of fuzzy soft topological space $\left(X, \tau_{\theta}, K\right)$ associated with $\breve{C}$ ech fuzzy soft closure space $(X, \theta, K)$ is studied. The fuzzy soft topological closure $\tau_{\theta}$-cl (respectively, interior $\tau_{\theta}$ - int) is defined and its relationship with the $\check{C}$ ech fuzzy soft closure operator $\theta$ (respectively, interior operator Int) is given (see Theorem 4). In Section 4, separation axioms $T_{0}$ and $T_{1}$ in $\check{C}$ ech fuzzy soft closure spaces are defined and their basic properties are discussed. Finally, in Section 5 , $T_{2} \check{C}$ ech fuzzy soft closure space and some other types of separation axioms, namely, semi- (respectively, pseudo and Uryshon) $T_{2}$ are defined. Some properties of each type are discussed and the relationship between aforementioned and $T_{0}$ (respectively, $T_{1}$ ) are given. Finally, several examples are given to support our study.

\section{PRELIMINARIES}

In this section we review some basic definitions and results related to fuzzy soft theory and $\check{C}$ ech fuzzy soft closure spaces that will be needed in the sequel, and we foresee the reader be familiar with the usual notions and most basic ideas of fuzzy set theory. Throughout our paper, $X$ will refer to the initial universe, $I=$ $[0,1], I_{0}=(0,1], I^{X}$ be the family of all fuzzy sets of $X$, and $K$ the set of parameters for $X$.

Definition 1. [11, 12] A fuzzy soft set (fss, for short) $\lambda_{\mathrm{A}}$ on $X$ is a mapping from $K$ to $I^{X}$, i.e., $\lambda_{\mathrm{A}}: K \rightarrow I^{X}$, where $\lambda_{\mathrm{A}}(h) \neq \overline{0}$ if $h \in A \subseteq K$ and $\lambda_{\mathrm{A}}(h)=\overline{0}$ if $h \notin A \subseteq K$, where $\overline{0}$ is the empty fuzzy set on $X$. The family of all fuzzy soft sets over $X$ denoted by $\mathcal{F}_{\text {ss }}(\mathrm{X}, K)$.

In the next definition, the basic operations between fuzzy soft sets are given.

Definition 2. [12] Let $\lambda_{\mathrm{A}}, \mu_{B} \in \mathcal{F}_{\mathrm{sS}}(\mathrm{X}, K)$, then

1. $\lambda_{\mathrm{A}}$ is called a fuzzy soft subset of $\mu_{B}$, denoted by $\lambda_{\mathrm{A}} \subseteq \mu_{B}$, if $\lambda_{\mathrm{A}}(h) \leq \mu_{B}(h)$, for all $h \in K$.

2. $\lambda_{\mathrm{A}}$ and $\mu_{B}$ are said to be equal, denoted by $\lambda_{\mathrm{A}}=\mu_{B}$ if $\lambda_{\mathrm{A}} \subseteq \mu_{B}$ and $\mu_{B} \subseteq \lambda_{A}$.

3. The union of $\lambda_{\mathrm{A}}$ and $\mu_{B}$, denoted by $\lambda_{\mathrm{A}} \cup \mu_{B}$ is the fss $\sigma_{(A \cup B)}(h)$ defined by $\sigma_{(A \cup B)}(h)=$ $\lambda_{A}(h) \vee \mu_{B}(h)$, for all $h \in K$.

4. The intersection of $\lambda_{\mathrm{A}}$ and $\mu_{B}$, denoted by $\lambda_{\mathrm{A}} \cap \mu_{B}$ is the fss $\sigma_{(A \cap B)}$ defined by $\sigma_{(A \cap B)}(h)=$ $\lambda_{\mathrm{A}}(h) \wedge \mu_{B}(h)$, for all $h \in K$.

Definition 3. [12] The null fss, denoted by $\overline{0}_{K}$, is a fss defined by $\overline{0}_{K}(h)=\overline{0}$, for all $h \in K$, where $\overline{0}$ is the empty fuzzy set of $X$.

Definition 4. [12] The universal fss, denoted by $\overline{1}_{K}$, is a fss defined by $\overline{1}_{K}(h)=\overline{1}$, for all $h \in K$, where $\overline{1}$ is the universal fuzzy set of $X$.

Definition 5. [12] The complement of a fss $\lambda_{\mathrm{A}} \in \mathcal{F}_{\mathrm{SS}}(\mathrm{X}, K)$, denoted $\overline{1}_{K}-\lambda_{\mathrm{A}}$, is the fss defined by $\left(\overline{1}_{K}-\lambda_{\mathrm{A}}\right)(h)=\overline{1}-\lambda_{\mathrm{A}}(h)$, for each $h \in K$, Its clear that $\overline{1}_{K}-\left(\overline{1}_{K}-\lambda_{\mathrm{A}}\right)=\lambda_{A}$.

Definition 6. [13] Two fss's $\lambda_{A}, \mu_{B} \in \mathcal{F}_{\text {ss }}(\mathrm{X}, K)$ are said to be disjoint, denoted by $\lambda_{A} \cap \mu_{B}=\overline{0}_{K}$, if $\lambda_{A}(h) \cap$ $\mu_{B}(h)=\overline{0}$ for all $h \in K$.

Definition 7. [14] A fuzzy soft set $\lambda_{A} \in \mathcal{F}_{S S}(X, K)$ is called fuzzy soft point, denoted by $x_{t}{ }^{h}$, if there exist $x \in X$ and $h \in K$ such that $\lambda_{\mathrm{A}}(h)(x)=t(0<t \leq 1)$ and 0 otherwise for all $y \in X-\{x\}$.

Definition 8. [14] The fuzzy soft point $x_{t}{ }^{h}$ is said to be belongs to the fss $\lambda_{A}$, denoted by $x_{t}{ }^{h} \widetilde{\in} \lambda_{\mathrm{A}}$ if for the element $h \in K, t \leq \lambda_{\mathrm{A}}(h)(x)$.

Definition 9. [15] Two fuzzy soft points $x_{t}^{h}$ and $y_{s}^{h^{\prime}}$ are said to be distinct if $x \neq y$ or $h \neq h^{\prime}$. 
Definition 10. [5,12] A fuzzy soft topological space(fsts, for short) $(X, \tau, K)$ where $X$ is a nonempty set with a fixed set of parameters and $\tau$ is a family of fuzzy soft sets over $X$ satisfying the following properties:

1. $\overline{0}_{K}, \overline{1}_{K} \in \tau$,

2. If $\lambda_{A}, \mu_{B} \in \tau$, then $\lambda_{A} \cap \mu_{B} \in \tau$,

3. If $\left(\lambda_{A}\right)_{i} \in \tau$, then $\bigcup_{i \in J \in}\left(\lambda_{A}\right)_{i} \in \tau$.

$\tau$ is called a topology of fuzzy soft sets on $X$. Every member of $\tau$ is called open fuzzy soft set (open-fss, for short). The complement of open-fss is called a closed fuzzy soft set (closed-fss, for short).

Definition 11. [9] An operator $\theta: \mathcal{F}_{S S}(X, K) \rightarrow \mathcal{F}_{S S}(X, K)$ is called $\check{C}$ ech fuzzy soft closure operator $(\check{C}$-fsco, for short) on $X$, if the following axioms are satisfied:

(C1) $\theta\left(\overline{0}_{K}\right)=\overline{0}_{K}$,

(C2) $\lambda_{\mathrm{A}} \subseteq \theta\left(\lambda_{A}\right)$, for all $\lambda_{A} \in \mathcal{F}_{\mathrm{SS}}(\mathrm{X}, K)$,

(C3) $\theta\left(\lambda_{A} \cup \mu_{B}\right)=\theta\left(\lambda_{A}\right) \cup \theta\left(\mu_{A}\right)$, for all $\lambda_{A}, \mu_{B} \in \mathcal{F}_{\text {ss }}(\mathrm{X}, K)$.

The triple $(X, \theta, K)$ is called a Cech fuzzy soft closure space ( $\breve{C} \mathcal{F}$-fscs, for short).

A fss $\lambda_{\mathrm{A}}$ is said to be closed-fss in $(X, \theta, K)$ if $\lambda_{\mathrm{A}}=\theta\left(\lambda_{A}\right)$. And a fss $\lambda_{\mathrm{A}}$ is said to be an open-fss if $\overline{1}_{K}-$ $\lambda_{A}$ is a closed-fss.

Proposition 1. [9] Let $(X, \theta, K)$ be a $\check{C} \mathcal{F}_{\text {-scs, }}$ and $\lambda_{\mathrm{A}}, \mu_{B} \in \mathcal{F}_{\mathrm{sS}}(\mathrm{X}, K)$ such that $\lambda_{\mathrm{A}} \subseteq \mu_{B}$, then $\theta\left(\lambda_{A}\right) \subseteq$ $\theta\left(\mu_{B}\right)$.

Definition 12. [9] Let $(X, \theta, K)$ be a $\check{C} \mathcal{F}$-scs, and let $\lambda_{\mathrm{A}} \in \mathcal{F}_{\mathrm{SS}}(\mathrm{X}, K)$. The interior of $\lambda_{\mathrm{A}}$, denoted by $\operatorname{Int}\left(\lambda_{\mathrm{A}}\right)$ is defined as $\operatorname{Int}\left(\lambda_{\mathrm{A}}\right)=\overline{1}_{K}-\left(\theta\left(\overline{1}_{K}-\lambda_{\mathrm{A}}\right)\right)$.

Proposition 2. [9] Let $(X, \theta, K)$ be a $\check{C} \mathcal{F}$-scs, and let $\lambda_{\mathrm{A}}, \mu_{B} \in \mathcal{F}_{\mathrm{SS}}(\mathrm{X}, K)$. Then

1. $\operatorname{Int}\left(\overline{0}_{K}\right)=\overline{0}_{K}$ and $\operatorname{Int}\left(\overline{1}_{K}\right)=\overline{1}_{K}$,

2. $\operatorname{Int}\left(\lambda_{A}\right) \subseteq \lambda_{A}$,

3. $\operatorname{Int}\left(\lambda_{A} \cap \mu_{B}\right)=\operatorname{Int}\left(\lambda_{A}\right) \cap \operatorname{Int}\left(\mu_{B}\right)$,

4. If $\lambda_{A} \subseteq \mu_{B}$, then $\operatorname{Int}\left(\lambda_{A}\right) \subseteq \operatorname{Int}\left(\mu_{B}\right)$,

5. $\lambda_{A}$ is an open-fss $\Leftrightarrow \operatorname{Int}\left(\lambda_{A}\right)=\lambda_{A}$,

6. $\operatorname{Int}\left(\lambda_{A}\right) \cup \operatorname{Int}\left(\mu_{B}\right) \subseteq \operatorname{Int}\left(\lambda_{A} \cup \mu_{B}\right)$.

Theorem 1. [9] Let $(X, \theta, K)$ be a $\check{C} \mathcal{F}$-scs and let $\tau_{\theta} \subseteq \mathcal{F}_{\text {ss }}(\mathrm{X}, K)$, defined as follows

$$
\tau_{\theta}=\left\{\overline{1}_{K}-\lambda_{\mathrm{A}}: \theta\left(\lambda_{\mathrm{A}}\right)=\lambda_{\mathrm{A}}\right\}
$$

Then $\tau_{\theta}$ is a fuzzy soft topology on $X$ and $\left(X, \tau_{\theta}, K\right)$ is called an associative fsts of $(X, \theta, K)$.

Definition 13. [9] Let $V$ be a non-empty subset of $X$, then $\bar{V}_{K}$ denotes the fuzzy soft set $V_{K}$ over $X$ for which $V(h)=\overline{1}_{V}$ for all $h \in K$, (where $\overline{1}_{V}: X \rightarrow I$ such that $\overline{1}_{V}(x)=1$ if $x \in V$ and $\overline{1}_{V}(x)=0$ if $x \notin V$ ).

Theorem 2. [9] Let $(X, \theta, K)$ be a $\check{C} \mathcal{F}$-scs, $V \subseteq X$ and let $\theta_{V}: \mathcal{F}_{s S}(V, K) \rightarrow \mathcal{F}_{S S}(V, K)$ defined as $\theta_{V}\left(\lambda_{\mathrm{A}}\right)=$ $\bar{V}_{K} \cap \theta\left(\lambda_{A}\right)$. Then $\theta_{V}$ is a $\check{C} \mathcal{F}$-sco. The triple $\left(V, \theta_{V}, K\right)$ is said to be $\check{C}$ ech fuzzy soft closure subspace $(\breve{C} \mathcal{F}$ sc subspace, for short) of $(X, \theta, K)$.

Definition 14. [9] Let $\theta_{1}$ and $\theta_{2}$ be two $\breve{C}$-fsco's on $X$. Then $\theta_{1}$ is said to finer than $\theta_{2}$, or equiventily $\theta_{2}$ is coarser than $\theta_{1}$, if for each $\lambda_{\mathrm{A}} \in \mathcal{F}_{\mathrm{SS}}(\mathrm{X}, K), \theta_{2}\left(\lambda_{\mathrm{A}}\right) \subseteq \theta_{1}\left(\lambda_{\mathrm{A}}\right)$.

\section{SOME PROPERTIES OF ASSOCIATIVE FUZZY SOFT TOPOLOGICAL SPACES}

In [10], Majeed and Maibed show that from each $\check{C} \mathcal{F}$-scs $(X, \theta, K)$ there exists an associated fsts $\left(X, \tau_{\theta}, K\right)$ (see Theorem 1). In this section we study the associated fsts $\left(X, \tau_{\theta}, K\right)$. Namely, we define the fuzzy soft topological closure $\tau_{\theta}$ - $c l$ (respectively, interior $\tau_{\theta^{-}}$int ) and study it is basic properties. In addition, we discuss the relation between the $\breve{C}$ ech fuzzy soft closure operator $\theta$ (respectively, interior operator $I n t$ ) and the fuzzy soft topological closure $\tau_{\theta}-c l$ (respectively, Interior $\tau_{\theta}$-int). 
Definition 15. Let $\left(X, \tau_{\theta}, K\right)$ be an associative fsts of $(X, \theta, K)$ and let $\lambda_{A} \in \mathcal{F}_{s S}(X, K)$. The fuzzy soft topological closure of $\lambda_{\mathrm{A}}$ with respect to $\theta$, denoted by $\tau_{\theta}-c l\left(\lambda_{A}\right)$, is the intersection of all closed fuzzy soft super sets of $\lambda_{A}$. i.e.,

$\tau_{\theta^{-}} \operatorname{cl}\left(\lambda_{A}\right)=\cap\left\{\rho_{C}: \lambda_{A} \subseteq \rho_{C}\right.$ and $\left.\theta\left(\rho_{C}\right)=\rho_{C}\right\}$.

From Theorem 1, it is clear that $\tau_{\theta}-\operatorname{cl}\left(\lambda_{A}\right)$ is the smallest closed-fss over $X$ which contains $\lambda_{A}$.

Proposition 3. Let $\left(\mathrm{X}, \tau_{\theta}, K\right)$ be an associative fsts of $(X, \theta, K)$ and let $\lambda_{A}, \mu_{B} \in \mathcal{F}_{S S}(X, K)$. Then

1. $\tau_{\theta^{-}} c l\left(\overline{0}_{K}\right)=\overline{0}_{K}$ and $\tau_{\theta}-c l\left(\overline{1}_{K}\right)=\overline{1}_{K}$,

2. $\lambda_{A} \subseteq \tau_{\theta}-\operatorname{cl}\left(\lambda_{A}\right)$,

3. If $\lambda_{A} \subseteq \mu_{B}$, then $\tau_{\theta^{-}} \operatorname{cl}\left(\lambda_{A}\right) \subseteq \tau_{\theta^{-}} \operatorname{cl}\left(\mu_{B}\right)$,

4. $\tau_{\theta^{-}} c l\left(\lambda_{A} \cup \mu_{B}\right)=\tau_{\theta^{-}} \operatorname{cl}\left(\lambda_{A}\right) \cup \tau_{\theta^{-}} c l\left(\mu_{B}\right)$,

5. $\tau_{\theta^{-}} \operatorname{cl}\left(\tau_{\theta^{-}} \operatorname{cl}\left(\lambda_{A}\right)\right)=\tau_{\theta^{-}} \operatorname{cl}\left(\lambda_{A}\right)$,

6. $\lambda_{A}$ is a closed-fss if and only if $\lambda_{A=} \tau_{\theta}-\operatorname{cl}\left(\lambda_{A}\right)$.

Proof. The proof of parts from 1 to 3 and 5 are follows directly from the Definition 15 . To prove part 4 , since $\lambda_{A} \subseteq \lambda_{A} \cup \mu_{B}$ and $\mu_{B} \subseteq \lambda_{A} \cup \mu_{B}$, then by 3 , we have $\tau_{\theta}-c l\left(\lambda_{A}\right) \subseteq \tau_{\theta}-c l\left(\lambda_{A} \cup \mu_{B}\right)$ and $\tau_{\theta}-c l\left(\mu_{B}\right) \subseteq$ $\tau_{\theta}-c l\left(\lambda_{A} \cup \mu_{B}\right)$.Thus, $\tau_{\theta^{-}}-c l\left(\lambda_{A}\right) \cup \tau_{\theta^{-}} \operatorname{cl}\left(\mu_{B}\right) \subseteq \tau_{\theta^{-}} \operatorname{cl}\left(\lambda_{A} \cup \mu_{B}\right)$. Conversely, by $2, \lambda_{A} \cup \mu_{B} \subseteq \tau_{\theta^{-}}$ $\operatorname{cl}\left(\lambda_{A}\right) \cup \tau_{\theta^{-}} \operatorname{cl}\left(\mu_{B}\right)$. Since $\tau_{\theta^{-}} \operatorname{cl}\left(\lambda_{A}\right)$ and $\tau_{\theta^{-}} \operatorname{cl}\left(\mu_{B}\right)$ are closed-fss. Then from Theorem 1, $\tau_{\theta^{-}} \operatorname{cl}\left(\lambda_{A}\right) \cup \tau_{\theta^{-}}$ $\operatorname{cl}\left(\mu_{B}\right)$ is the smallest closed-fss contains $\lambda_{A} \cup \mu_{B}$. But $\tau_{\theta}$-cl $\left(\lambda_{A} \cup \mu_{B}\right)$ is the smallest closed-fss contains $\lambda_{A} \cup \mu_{B}$, this implies $\tau_{\theta}$-cl $\left(\lambda_{A} \cup \mu_{B}\right) \subseteq \tau_{\theta}$-cl $\left(\lambda_{A}\right) \cup \tau_{\theta}$-cl $\left(\mu_{B}\right)$. Hence, we obtain the equality. Finally, to prove 6 , let $\lambda_{A}$ be a closed-fss. By 2, we get $\lambda_{A} \subseteq \tau_{\theta^{-}} \operatorname{cl}\left(\lambda_{A}\right)$ and $\tau_{\theta^{-}} \operatorname{cl}\left(\lambda_{A}\right)$ is the smallest closed-fss which contains $\lambda_{A}$ and since $\lambda_{A}$ is a closed-fss, then $\tau_{\theta^{-}} \operatorname{cl}\left(\lambda_{A}\right) \subseteq \lambda_{A}$. Hence, $\lambda_{A}=\tau_{\theta^{-}}$ $\operatorname{cl}\left(\lambda_{A}\right)$. Conversely, suppose that $\lambda_{A}=\tau_{\theta}-c l\left(\lambda_{A}\right)$. Since $\tau_{\theta}-\operatorname{cl}\left(\lambda_{A}\right)$ is the closed-fss, then $\lambda_{A}$ is a closedfss.

Now, we introduce the definition of fuzzy soft interior of a fss in the associative fsts of $\left(\mathrm{X}, \tau_{\theta}, K\right)$ and give some properties of it.

Definition 16. Let $\left(\mathrm{X}, \tau_{\theta}, K\right)$ be an associative fsts of $(X, \theta, K)$ and let $\lambda_{A} \in \mathcal{F}_{S S}(X, K)$. The fuzzy soft topological interior of $\lambda_{A}$ with respect to $\theta$, denoted by $\tau_{\theta}-\operatorname{int}\left(\lambda_{A}\right)$ is the union of all open fuzzy soft subset of $\lambda_{A}$. i.e.,

$\tau_{\theta^{-}} \operatorname{int}\left(\lambda_{A}\right)=\bigcup\left\{\rho_{C}: \rho_{C} \subseteq \lambda_{A}\right.$ and $\left.\theta\left(\overline{1}_{K}-\rho_{C}\right)=\overline{1}_{K}-\rho_{C}\right\}$

Clearly, $\tau_{\theta}-\operatorname{int}\left(\lambda_{A}\right)$ is the largest open-fss contained in $\lambda_{A}$.

Proposition 4. Let $\left(\mathrm{X}, \tau_{\theta}, K\right)$ be an associative fuzzy soft topological space of $(X, \theta, K)$ and let $\lambda_{A}, \mu_{B} \in$ $\mathcal{F}_{S S}(X, K)$. Then

1. $\tau_{\theta^{-}} \operatorname{int}\left(\overline{0}_{K}\right)=\overline{0}_{K}$ and $\tau_{\theta^{-}} \operatorname{int}\left(\overline{1}_{K}\right)=\overline{1}_{K}$,

2. $\tau_{\theta}-\operatorname{int}\left(\lambda_{A}\right) \subseteq \lambda_{A}$

3. If $\lambda_{A} \subseteq \mu_{B}$, then $\tau_{\theta^{-}} \operatorname{int}\left(\lambda_{A}\right) \subseteq \tau_{\theta^{-}} \operatorname{int}\left(\mu_{B}\right)$,

4. $\tau_{\theta}$-int $\left(\lambda_{A} \cap \mu_{B}\right)=\tau_{\theta}$-int $\left(\lambda_{A}\right) \cap \tau_{\theta}$-int $\left(\mu_{B}\right)$,

5. $\tau_{\theta}-\operatorname{int}\left(\tau_{\theta}-\operatorname{int}\left(\lambda_{A}\right)\right)=\tau_{\theta}-\operatorname{int}\left(\lambda_{A}\right)$

6. $\lambda_{A}$ is an open-fss if and only if $\lambda_{A}=\tau_{\theta}$-int $\left(\lambda_{A}\right)$.

Proof. The prove is similar to the proof of Proposition 3.

Theorem 3. Let $\left(\mathrm{X}, \tau_{\theta}, K\right)$ be an associative fsts of $(X, \theta, K)$ and $\lambda_{A} \in \mathcal{F}_{S S}(X, K)$. Then

1. $\overline{1}_{K}-\left(\tau_{\theta}-\operatorname{int}\left(\lambda_{A}\right)\right)=\tau_{\theta}-\operatorname{cl}\left(\overline{1}_{K}-\lambda_{A}\right)$,

2. $\overline{1}_{K}-\left(\tau_{\theta}-\operatorname{cl}\left(\lambda_{A}\right)\right)=\tau_{\theta}-\operatorname{int}\left(\overline{1}_{K}-\lambda_{A}\right)$.

Proof. The proof is obtained by using Proposition 4 part 2, and Proposition 3 part 3. 
In the next theorem, we discuss the relationship between the $\breve{C}$ ech fuzzy soft closure operator $\theta$ (respectively, interior operator Int) and the fuzzy soft topological closure $\tau_{\theta^{-}}$cl (respectively, Interior $\tau_{\theta^{-}}$ int) for any fss $\lambda_{A} \in \mathcal{F}_{S S}(X, K)$.

Theorem 4. Let $(\mathrm{X}, \theta, K)$ be $\check{\mathrm{C}} \mathcal{F}$-scs and $\left(\mathrm{X}, \tau_{\theta}, K\right)$ be an associative fuzzy soft topological space of $(X, \theta, K)$. Then for any fss $\lambda_{A} \in \mathcal{F}_{S S}(X, K)$

$$
\tau_{\theta}-\operatorname{int}\left(\lambda_{A}\right) \subseteq \operatorname{Int}\left(\lambda_{A}\right) \subseteq \lambda_{A} \subseteq \theta\left(\lambda_{A}\right) \subseteq \tau_{\theta}-\operatorname{cl}\left(\lambda_{A}\right) \text {. }
$$

Proof. First, we prove $\tau_{\theta}$-int $\left(\lambda_{A}\right) \subseteq \operatorname{Int}\left(\lambda_{A}\right) \subseteq \lambda_{A}$. Since $\tau_{\theta}$-int $\left(\lambda_{A}\right) \subseteq \lambda_{A}$, then by 4 of Proposition 2 , $\operatorname{Int}\left(\tau_{\theta}-\operatorname{int}\left(\lambda_{A}\right)\right) \subseteq \operatorname{Int}\left(\lambda_{A}\right)$. But $\tau_{\theta}$-int $\left(\lambda_{A}\right)$ is an open-fss, this implies $\tau_{\theta}$-int $\left(\lambda_{A}\right) \subseteq \operatorname{Int}\left(\lambda_{A}\right)$, and from the definition of $\breve{C}$ ech fuzzy soft interior operator, we get $\tau_{\theta}$-int $\left(\lambda_{A}\right) \subseteq \operatorname{Int}\left(\lambda_{A}\right) \subseteq \lambda_{A}$.

Now, to prove $\lambda_{A} \subseteq \theta\left(\lambda_{A}\right) \subseteq \tau_{\theta}-\operatorname{cl}\left(\lambda_{A}\right)$. Since $\lambda_{A} \subseteq \tau_{\theta}-\operatorname{cl}\left(\lambda_{A}\right)$ and $\tau_{\theta}$-cl $\left(\lambda_{A}\right)$ is a closed-fss, then $\theta\left(\lambda_{A}\right) \subseteq \theta\left(\tau_{\theta^{-}} \operatorname{cl}\left(\lambda_{A}\right)\right)=\tau_{\theta}-c l\left(\lambda_{A}\right)$ and by (C2) of Definition 11, we have $\lambda_{A} \subseteq \theta\left(\lambda_{A}\right) \subseteq \tau_{\theta^{-}} c l\left(\lambda_{A}\right)$. Hence, we obtain the required result.

Remark 1. In the above theorem the equality hold for the part $\tau_{\theta}$-int $\left(\lambda_{A}\right) \subseteq \operatorname{int}\left(\lambda_{A}\right) \subseteq \lambda_{A}$ (respectively, $\left.\lambda_{A} \subseteq \theta\left(\lambda_{A}\right) \subseteq \tau_{\theta}-c l\left(\lambda_{A}\right)\right)$ if $\lambda_{A}$ is an open- (respectively, closed-) fss in $(X, \theta, K)$. But in general, the equality of the above theorem is not true, so we give an example to explain that.

Example 1. Let $X=\{a, b, c\}, K=\left\{h_{1}, h_{2}\right\}$. Define $\mu_{B} \in \mathcal{F}_{S S}(X, K)$ such that $\mu_{B}=\left\{\left(h_{1}, b_{0.5}\right),\left(h_{2}, b_{0.5}\right)\right\}$. Define $\breve{C}$-fsco $\theta: \mathcal{F}_{S S}(X, K) \rightarrow \mathcal{F}_{S S}(X, K)$ as follows:

$$
\theta\left(\lambda_{A}\right)= \begin{cases}\overline{0}_{K} & \text { if } \lambda_{A}=\overline{0}_{K} \\ \left\{\left(h_{1}, a_{0.5} \vee b_{0.5}\right),\left(h_{2}, a_{0.5} \vee b_{0.5}\right)\right\} & \text { if } \lambda_{A} \subseteq \mu_{B} \\ \overline{1}_{K} & \text { otherwise }\end{cases}
$$

It is clear that $\theta$ satisfy the axioms (C1), (C2) and (C3) of Definition 11, in addition $\theta\left(\mu_{B}\right) \neq \theta\left(\theta\left(\mu_{B}\right)\right)$. Thus, $(\mathrm{X}, \theta, K)$ is a $\breve{\mathcal{F}} \mathcal{F}$-scs. The associative fuzzy soft topology $\tau_{\theta}$ of $(\mathrm{X}, \theta, K)$ is $\left\{\overline{0}_{K}, \overline{1}_{K}\right\}$. Now, consider the fss $\lambda_{A}=\left\{\left(h_{1}, a_{1} \vee b_{0.7} \vee c_{1}\right),\left(h_{2}, a_{1} \vee b_{0.9} \vee c_{1}\right)\right\}$. Then, $\operatorname{Int}\left(\lambda_{A}\right)=\left\{\left(h_{1}, a_{0.5} \vee b_{0.5} \vee c_{1}\right),\left(h_{2}, a_{0.5} \vee\right.\right.$ $\left.\left.b_{0.5} \vee c_{1}\right)\right\}$ and $\tau_{\theta}$-int $\left(\lambda_{A}\right)=\overline{0}_{K}$. Hence, $\operatorname{Int}\left(\lambda_{A}\right) \nsubseteq \tau_{\theta}-\operatorname{int}\left(\lambda_{A}\right)$. On the other hand consider $\lambda_{A}=$ $\left\{\left(h_{1}, b_{0.4}\right),\left(h_{2}, b_{0.2}\right)\right\}$. Then $\tau_{\theta}$-cl $\left.\left(\lambda_{A}\right)\right)=\overline{1}_{K} \nsubseteq\left\{\left(h_{1}, a_{0.5} \vee b_{0.5}\right),\left(h_{2}, a_{0.5} \vee b_{0.5}\right)\right\}=\theta\left(\lambda_{A}\right)$. Thus, $\tau_{\theta^{-}}$ $\left.\operatorname{cl}\left(\lambda_{A}\right)\right) \nsubseteq \theta\left(\lambda_{A}\right)$.

\section{4. $T_{i}$-ČECH FUZZY SOFT CLOSURE SPACES, $i=0,1$}

This section is devoted to defining separation axioms $\mathrm{T}_{0}$ and $\mathrm{T}_{1}$ in $\check{C} \mathcal{F}$-scs's and its associated fsts's. We discuss the relation between $\mathrm{T}_{0}$ and $\mathrm{T}_{1}$, and study the hereditary property on $\breve{C} \mathcal{F}$-scs's. Also, we give the relation between $\breve{C} \mathcal{F}$-scs $(X, \theta, K)$ and its associated fsts $\left(X, \tau_{\theta}, K\right)$ when $\left(X, \tau_{\theta}, K\right)$ is $\mathrm{T}_{\mathrm{i}}, i=0,1$.

Definition 17. A $\check{C} \mathcal{F}$-scs $(X, \theta, K)$ is said to be $\mathrm{T}_{0}-\check{C} \mathcal{F}$-scs, if for every pair of distinct fuzzy soft points $x_{t}^{h}$ and $y_{s}^{h^{\prime}}$, either $x_{t}^{h} \widetilde{\notin} \theta\left(y_{s}^{h^{\prime}}\right)$ or $y_{s}^{h^{\prime}} \widetilde{\notin} \theta\left(x_{t}^{h}\right)$.

Now we give some examples to illustrate Definition 17.

Example 2. Let $(X, \theta, K)$ be the discrete $\check{C} \mathcal{F}$-scs (i.e., $\theta\left(\lambda_{A}\right)=\lambda_{A}$ for all $\left.\lambda_{A} \in \mathcal{F}_{\text {sS }}(X, K)\right)$, then $(X, \theta, K)$ is a $\mathrm{T}_{0}-\check{C} \mathcal{F}$-scs.

Example 3. Let $(X, \theta, K)$ be the trivial $\breve{C} \mathcal{F}$-scs (i.e., $\theta\left(\lambda_{A}\right)=\overline{1}_{K}$ for all $\left.\lambda_{A} \in \mathcal{F}_{\text {ss }}(\mathrm{X}, K)\right)$, then $(X, \theta, K)$ is

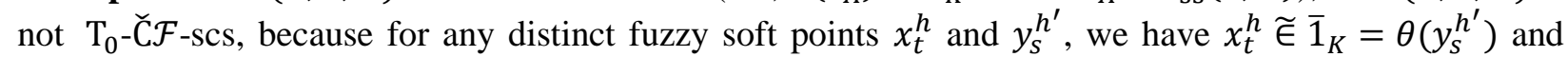
$y_{s}^{h^{\prime}} \widetilde{\in} \overline{1}_{K}=\theta\left(x_{t}^{h}\right)$. 
Example 4. Let $X=\{a, b\}, \mathrm{K}=\left\{h_{1}, h_{2}\right\}$ and let $\lambda_{A}^{*} \subseteq \mathcal{F}_{\text {SS }}(\mathrm{X}, K)$ such that $\lambda_{A}^{*}=\left\{\left(h_{1}, a_{t_{1}} \vee b_{s_{1}}\right),\left(h_{2}, a_{t_{2}} \vee b_{s_{2}}\right)\right.$; $\left.t_{1}, t_{2}, s_{1}, s_{2} \in I_{0}\right\}$. Define $\theta: \mathcal{F}_{\text {ss }}(\mathrm{X}, K) \rightarrow \mathcal{F}_{\text {ss }}(\mathrm{X}, K)$ as follows:

$$
\theta\left(\lambda_{A}\right)= \begin{cases}\overline{0}_{K} & \text { if } \lambda_{A}=\overline{0}_{K}, \\ a_{1}^{h_{1}} & \text { if } \lambda_{A} \in\left\{a_{t_{1}}^{h_{1}} ; t_{1} \in I_{0}\right\}, \\ a_{1}^{h_{2}} & \text { if } \lambda_{A} \in\left\{a_{t_{2}}^{h_{2}} ; t_{2} \in I_{0}\right\}, \\ b_{s_{1+0.2}}^{h_{1}} & \text { if } \lambda_{A} \in\left\{b_{s_{1}}^{h_{1}} ; 0<s_{1}<0.8\right\}, \\ b_{1}^{h_{1}} & \text { if } \lambda_{A} \in\left\{b_{s_{1}}^{h_{1}} ; 0.8 \leq s_{1} \leq 1\right\}, \\ \left\{\left(h_{1}, \theta\left(a_{t_{1}}^{h_{1}}\right) \cup \theta\left(b_{s_{1}}^{h_{1}}\right)\right),\left(h_{2}, \theta\left(a_{t_{2}}^{h_{2}}\right) \cup \theta\left(b_{s_{2}}^{h_{2}}\right)\right)\right\} \quad \text { if } \lambda_{A} \in \lambda_{A}^{*} .\end{cases}
$$

Then $(X, \theta, K)$ is a $\check{C} \mathcal{F}$-scs. To show $(X, \theta, K)$ is $\mathrm{T}_{0}$ - $\check{C} \mathcal{F}$-scs, we have three cases for distinct fuzzy soft points in $X$.

$\underline{\operatorname{Case}(1)}$. If $a \neq b$ and $h_{1}=h_{2}$, then we have $a_{t_{1}}^{h_{1}}$ and $b_{s_{1}}^{h_{1}}$ are distinct fuzzy soft points. It is clear that $b_{s_{1}}^{h_{1}}$ $\widetilde{\notin} \theta\left(a_{t_{1}}^{h_{1}}\right)$ because $s_{1}>\left(a_{1}^{h_{1}}\right)\left(h_{1}\right)(b)=\left(a_{1}\right)(b)=0$. Similarly, $a_{t_{1}}^{h_{2}}$ and $b_{s_{1}}^{h_{2}}$ are distinct fuzzy soft points and $b_{s_{1}}^{h_{2}} \widetilde{\notin} \theta\left(a_{t_{1}}^{h_{2}}\right)$.

Case(2). If $a=b$ and $h_{1} \neq h_{2}$, then $a_{t_{1}}^{h_{1}}$ and $a_{t_{2}}^{h_{2}}$ are distinct fuzzy soft points. It is clear that $a_{t_{1}}^{h_{1}} \widetilde{\notin} \theta\left(a_{t_{2}}^{h_{2}}\right)$ because $t_{1}>\theta\left(a_{1}^{h_{2}}\right)\left(h_{1}\right)(a)=\overline{0}(a)=0$. Similarly, $b_{s_{1}}^{h_{1}}$ and $b_{s_{2}}^{h_{2}}$ are distinct fuzzy soft points and $b_{s_{2}}^{h_{2}}$ $\widetilde{\notin} \theta\left(b_{s_{1}}^{h_{1}}\right)$.

Case(3). If $a \neq b$ and $h_{1} \neq h_{2}$, then we have $a_{t}^{h_{1}}$ and $b_{s}^{h_{2}}$ are distinct fuzzy soft points such that $b_{s}^{h_{2}}$ $\widetilde{\notin} \theta\left(a_{t}^{h_{1}}\right)$. Similarly, $a_{t}^{h_{2}}$ and $b_{s}^{h_{1}}$ are distinct fuzzy soft points and $b_{s}^{h_{1}} \widetilde{\notin} \theta\left(a_{t}^{h_{2}}\right)$. Hence, $(X, \theta, K)$ is $\mathrm{T}_{0^{-}}$ $\check{\mathrm{C}} \mathcal{F}$-scs.

Theorem 5. Let $(X, \theta, K)$ be a $T_{0}$ - $\check{C} \mathcal{F}$-scs, then for any two distinct fuzzy soft points $x_{t}^{h}$ and $y_{s}^{h^{\prime}}, \theta\left(x_{t}^{h}\right) \neq$ $\theta\left(y_{s}^{h^{\prime}}\right)$.

Proof. Let $(X, \theta, K)$ be a $T_{0}$-Č $\mathcal{F}$-scs, and let $x_{t}^{h}$ and $y_{s}^{h^{\prime}}$ be any two distinct fuzzy soft points. Suppose that $\theta\left(x_{t}^{h}\right)=\theta\left(y_{s}^{h^{\prime}}\right)$. Since $x_{t}^{h} \widetilde{\in} \theta\left(x_{t}^{h}\right)$ and $y_{s}^{h^{\prime}} \widetilde{\in} \theta\left(y_{s}^{h^{\prime}}\right)$. Then from hypothesis, $x_{t}^{h} \widetilde{\in} \theta\left(y_{s}^{h^{\prime}}\right)$ and $y_{s}^{h^{\prime}} \widetilde{\in} \theta\left(x_{t}^{h}\right)$. This implies $(X, \theta, K)$ is not $T_{0}-\check{C} \mathcal{F}$-scs, which is a contradiction. Hence, $\theta\left(x_{t}^{h}\right) \neq \theta\left(y_{s}^{h^{\prime}}\right)$

The converse of above theorem is not true, as the following example show.

Example 5. Let $X=\{a, b\}, K=\{h\}$. Define $\theta: \mathcal{F}_{\mathrm{sS}}(\mathrm{X}, K) \rightarrow \mathcal{F}_{\mathrm{sS}}(\mathrm{X}, K)$ as follows:

$$
\theta\left(\lambda_{A}\right)=\left\{\begin{array}{cl}
\overline{0}_{K} & \text { if } \lambda_{A}=\overline{0}_{K}, \\
\left\{\left(h, a_{0.2} \vee b_{0.1}\right)\right\} & \text { if } \lambda_{A} \subseteq a_{0.2}^{h}, \\
\left\{\left(h, a_{0.1} \vee b_{0.2}\right)\right\} & \text { if } \lambda_{A} \subseteq b_{0.2}^{h}, \\
\lambda_{A} & \text { if } \lambda_{A} \in\left\{a_{t}^{h} ; 0.2<t \leq 1\right\}, \\
\lambda_{A} & \text { if } \lambda_{A} \in\left\{b_{s}^{h} ; 0.2<s \leq 1\right\}, \\
\theta\left(a_{t}^{h}\right) \cup \theta\left(b_{s}^{h}\right) & \text { if } \lambda_{A} \in\left\{\left(h, a_{t} \vee b_{s}\right) ; t, s \in I_{0}\right\} .
\end{array}\right.
$$

Then $(X, \theta, K)$ is $\check{C} \mathcal{F}$-scs. It is clear that from the definition of $\theta, \theta\left(a_{t}^{h}\right) \neq \theta\left(b_{s}^{h}\right)$ for any two-distinct fuzzy soft points $a_{t}^{h}$ and $b_{s}^{h}$. However, $(X, \theta, K)$ is not $T_{0}$ - $\breve{C} \mathcal{F}$-scs, since there exist two distinct fuzzy soft point $a_{0.1}^{h}$ and $b_{0.1}^{h}$ such that $a_{0.1}^{h} \widetilde{\in} \theta\left(b_{0.1}^{h}\right)$ and $b_{0.1}^{h} \widetilde{\in} \theta\left(a_{0.1}^{h}\right)$.

Next, we show that $T_{0}$ is hereditary property on $\check{C} \mathcal{F}$-scs's.

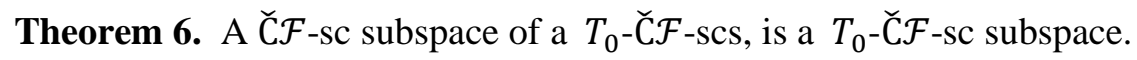


Proof. Let $(X, \theta, K)$ be a $T_{0}$ - $\breve{\mathcal{F}} \mathcal{F}$-scs and $\left(V, \theta_{V}, K\right)$ be a $\breve{C} \mathcal{F}$-sc subspace of $(X, \theta, K)$. Let $x_{t}^{h}$ and $y_{s}^{h^{\prime}}$ be any two-distinct fuzzy soft points in $\left(V, \theta_{V}, K\right)$. Since $\mathcal{F}_{\text {SS }}(V, K) \subseteq \mathcal{F}_{\text {SS }}(X, K)$, then $x_{t}^{h}$ and $y_{s}^{h^{\prime}}$ are distinct fuzzy soft points in $\mathcal{F}_{\mathrm{sS}}(\mathrm{X}, K)$. Since $(X, \theta, K)$ is $T_{0}$ - $\check{C}$-fscs. Then $x_{t}^{h} \widetilde{\notin} \theta\left(y_{s}^{h^{\prime}}\right)$ or $y_{s}^{h^{\prime}} \widetilde{\notin} \theta\left(x_{t}^{h}\right)$. This implies either $x_{t}^{h} \widetilde{\notin} \theta\left(y_{s}^{h^{\prime}}\right) \cap \bar{V}_{K}$ or $y_{s}^{h^{\prime}} \widetilde{\notin} \theta\left(x_{t}^{h}\right) \cap \bar{V}_{K}$. Then $x_{t}^{h} \widetilde{\notin} \theta_{V}\left(y_{s}^{h^{\prime}}\right)$ or $y_{s}^{h^{\prime}} \widetilde{\notin} \theta_{V}\left(x_{t}^{h}\right)$. Hence $\left(V, \theta_{V}, K\right)$ is $T_{0}-\check{C} \mathcal{F}$-sc subspace.

Definition 18. An associative fsts $\left(X, \tau_{\theta}, K\right)$ of $\check{C} \mathcal{F}$-fscs $(X, \theta, K)$ is said to be $\mathrm{T}_{0}$-fsts, if for every two distinct fuzzy soft points $x_{t}^{h}$ and $y_{s}^{h^{\prime}}$, either $x_{t}^{h} \widetilde{\notin} \tau_{\theta}-c l\left(y_{s}^{h^{\prime}}\right)$ or $y_{s}^{h^{\prime}} \widetilde{\notin} \tau_{\theta}-c l\left(x_{t}^{h}\right)$.

The next theorem give the relationship between the associative fsts $\left(X, \tau_{\theta}, K\right)$ which is $\mathrm{T}_{0}$-fsts and $\check{C} \mathcal{F}$-fscs $(X, \theta, K)$.

Theorem 7. If $\left(X, \tau_{\theta}, K\right)$ is a $T_{0}$-fsts, then $(X, \theta, K)$ is also $T_{0}$ - $\check{C} \mathcal{F}$-scs.

Proof. Let $x_{t}^{h}$ and $y_{s}^{h^{\prime}}$ be any two distinct fuzzy soft point in $(X, \theta, K)$. Since $\left(X, \tau_{\theta}, K\right)$ is a $T_{0}$-fsts, then $x_{t}^{h} \widetilde{\notin} \tau_{\theta}-c l\left(y_{s}^{h^{\prime}}\right)$ or $y_{s}^{h^{\prime}} \widetilde{\notin} \tau_{\theta}$-cl( $\left.x_{t}^{h}\right)$. By Theorem 4 we get, $x_{t}^{h} \widetilde{\notin} \theta\left(y_{s}^{h^{\prime}}\right)$ or $y_{s}^{h^{\prime}} \widetilde{\notin} \theta\left(x_{t}^{h}\right)$. This implies $(X, \theta, K)$ is $T_{0}-\check{C} \mathcal{F}$-scs.

The converse Theorem 7 is not true, as we shown in the following example.

Example 6. Let $X=\{a, b\}, K=\left\{h_{1}, h_{2}\right\}$. Define $\theta: \mathcal{F}_{\mathrm{sS}}(\mathrm{X}, K) \rightarrow \mathcal{F}_{\mathrm{sS}}(\mathrm{X}, K)$ as follows:

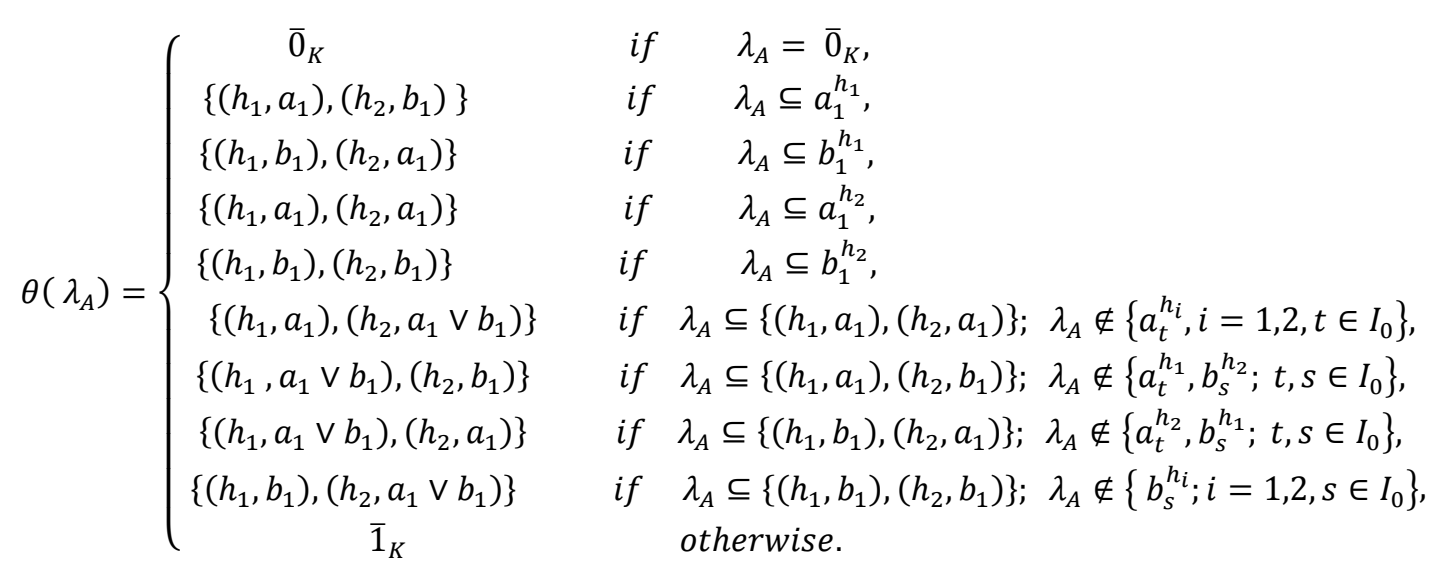

Then $(X, \theta, K)$ is $T_{0}$ - $\breve{C} \mathcal{F}$-scs. But $\left(X, \tau_{\theta}, K\right)$ is not $T_{0}$-fsts, because $\tau_{\theta}=\left\{\overline{1}_{K}-\lambda_{A}: \theta\left(\lambda_{A}\right)=\lambda_{A}\right\}=\left\{\overline{0}_{K}, \overline{1}_{K}\right\}$.

Definition 19. A $\check{C}$-fscs $(X, \theta, K)$ is said to be $\mathrm{T}_{1}-\check{C} \mathcal{F}$-scs, if for every two distinct fuzzy soft points $x_{t}^{h}$ and $y_{s}^{h^{\prime}}$ we have $x_{t}^{h} \widetilde{\notin} \theta\left(y_{s}^{h^{\prime}}\right)$ and $y_{s}^{h^{\prime}} \widetilde{\notin} \theta\left(x_{t}^{h}\right)$.

Example 7. Let $X=\{a, b\}, K=\left\{h_{1}, h_{2}\right\}$, and let $\lambda_{A}^{*} \subseteq \mathcal{F}_{\text {sS }}(X, K)$ such that $\lambda_{A}^{*}=\left\{\left(h_{1}, a_{t_{1}} \vee b_{s_{1}}\right),\left(h_{2}, a_{t_{2}} \vee b_{s_{2}}\right)\right.$; $\left.t_{1}, t_{2}, s_{1}, s_{2} \in I_{0}\right\}$. Define $\theta: \mathcal{F}_{\text {ss }}(\mathrm{X}, K) \rightarrow \mathcal{F}_{\text {ss }}(\mathrm{X}, K)$ as follows:

$$
\theta\left(\lambda_{A}\right)= \begin{cases}\overline{0}_{K} & \text { if } \lambda_{A}=\overline{0}_{K}, \\ a_{1}^{h_{1}} & \text { if } \lambda_{A} \subseteq a_{1}^{h_{1}}, \\ a_{1}^{h_{2}} & \text { if } \lambda_{A} \subseteq a_{1}^{h_{2}}, \\ b_{1}^{h_{1}} & \text { if } \lambda_{A} \subseteq b_{1}^{h_{1}}, \\ b_{0.9}^{h_{2}} & \text { if } \lambda_{A} \in\left\{b_{s}^{h_{2}}, 0<s<0.9\right\}, \\ b_{1}^{h_{2}} & \text { if } \lambda_{A} \in\left\{b_{s}^{h_{2}}, 0.9 \leq s \leq 1\right\}, \\ \left\{\left(h_{1}, \theta\left(a_{t_{1}}^{h_{1}}\right) \cup \theta\left(b_{s_{1}}^{h_{1}}\right)\right),\left(h_{2}, \theta\left(a_{t_{2}}^{h_{2}}\right) \cup \theta\left(b_{s_{2}}^{h_{2}}\right)\right)\right\} & \text { if } \lambda_{A} \in \lambda_{A}^{*} .\end{cases}
$$


Then $(X, \theta, K)$ is $T_{1}$-ᄃ̌ $\mathcal{F}$-scs. Since for any two distinct fuzzy soft points $a_{t}^{h}$ and $b_{s}^{h^{\prime}}$ we have $a_{t}^{h} \widetilde{\notin} \theta\left(b_{s}^{h^{\prime}}\right)$ and $b_{s}^{h^{\prime}} \widetilde{\notin} \theta\left(a_{t}^{h}\right)$.

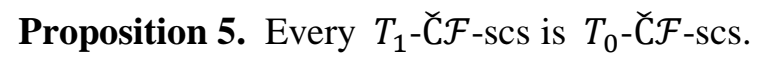

Proof. Follows directly from the definition of $T_{1}$ - $\check{\mathcal{F}}$-scs.

The converse of Proposition 5 is not true, as seen in the following example.

Example 8. Let $X=\{a, b\}, K=\{h\}$. Define $\theta: \mathcal{F}_{\text {sS }}(\mathrm{X}, K) \rightarrow \mathcal{F}_{\mathrm{SS}}(\mathrm{X}, K)$ as follows:

$$
\theta\left(\lambda_{A}\right)= \begin{cases}\overline{0}_{K} & \text { if } \lambda_{A}=\overline{0}_{K}, \\ a_{t+0.1}^{h} & \text { if } \lambda_{A} \in\left\{a_{t}^{h}, 0<t<0.9\right\}, \\ a_{1}^{h} & \text { if } \lambda_{A} \in\left\{a_{t}^{h}, 0.9 \leq t \leq 1\right\}, \\ \overline{1}_{K} & \text { otherwise. }\end{cases}
$$

Then $(X, \theta, K)$ is $\mathrm{T}_{0}-\check{C} \mathcal{F}$-scs. Since for any two distinct fuzzy soft points $a_{t}^{h}$ and $b_{s}^{h}, \mathrm{t}, \mathrm{s} \in(0,1]$. We have, $b_{s}^{h} \widetilde{\notin} \theta\left(a_{t}^{h}\right)$. However, it is not $T_{1}$ - ̌C $\mathcal{F}$-scs because $a_{t}^{h} \widetilde{\in} \theta\left(b_{s}^{h}\right)=\overline{1}_{K}$.

Theorem 8. If every fuzzy soft point in a $\check{C} \mathcal{F}$-scs $(X, \theta, K)$ is closed-fss, then $(X, \theta, K)$ is $T_{1}$ - $\check{C} \mathcal{F}$-scs.

Proof: Let $x_{t}^{h}$ and $y_{s}^{h^{\prime}}$ be any two distinct fuzzy soft points in $(X, \theta, K)$. From hypothesis, we have $\theta\left(x_{t}^{h}\right)=x_{t}^{h}$ and $\theta\left(y_{s}^{h^{\prime}}\right)=y_{s}^{h^{\prime}}$. This implies $x_{t}^{h} \widetilde{\notin} \theta\left(y_{s}^{h^{\prime}}\right)$ and $y_{s}^{h^{\prime}} \widetilde{\notin} \theta\left(x_{t}^{h}\right)$. Thus, $(X, \theta, K)$ is a $T_{1}$ - ᄃ́F scs.

The converse of above theorem is not true in general as we seen in the following example.

Example 9. In Example 7, $(X, \theta, K)$ is $T_{1}$ - $\breve{C} \mathcal{F}$-scs, yet there exists fuzzy soft point $b_{o .6}^{h_{2}}$ such that $\theta\left(b_{o .6}^{h_{2}}\right)=$ $b_{o .9}^{h_{2}}$.

Theorem 9. A $\check{C}$-fsc subspace of $T_{1}$ - $\breve{C} \mathcal{F}$-scs is $T_{1}$ - $\check{C} \mathcal{F}$-sc subspace.

Proof. Similar to the proof of Theorem 6.

Definition 20. An associative fsts $\left(X, \tau_{\theta}, K\right)$ of $\check{C} \mathcal{F}$-scs, $(X, \theta, K)$ is said to be $\mathrm{T}_{1}$-fsts, if for every two distinct fuzzy soft points $x_{t}^{h}$ and $y_{s}^{h^{\prime}}$, we have $x_{t}^{h} \widetilde{\notin} \tau_{\theta}$-cl $\left(y_{s}^{h^{\prime}}\right)$ and $y_{s}^{h^{\prime}} \widetilde{\notin} \tau_{\theta}-c l\left(x_{t}^{h}\right)$.

Theorem 10. If $\left(X, \tau_{\theta}, K\right)$ is a $T_{1}$-fsts, then $(X, \theta, K)$ is also $T_{1}$ - $\check{C} \mathcal{F}$-scs.

Proof. Similar to the proof of Theorem 7.

Proposition 6. If $\left(X, \theta_{1}, K\right)$ is $T_{i}$ - $\check{C} \mathcal{F}$-scs and $\theta_{2}$ is a $\check{C}$-fsco on $X$ such that $\theta_{2}$ is coarser than $\theta_{1}$, then $\left(X, \theta_{2}, K\right)$ is $T_{i^{-}}$С̆ $\mathcal{F}$-scs, $i=0,1$.

Proof. We prove the proposition when $i=1$, and the proof is similar for $i=0$. Let $x_{t}^{h}$ and $y_{s}^{h^{\prime}}$ be any two distinct fuzzy soft points in $\left(X, \theta_{2}, K\right)$. From hypothesis $\left(X, \theta_{1}, K\right)$ is $T_{1}$ - ᄃ̌ $\mathcal{F}$-scs, this yield $x_{t}^{h} \widetilde{\notin} \theta_{1}\left(y_{s}^{h^{\prime}}\right)$ and $y_{s}^{h^{\prime}} \widetilde{\notin} \theta_{1}\left(x_{t}^{h}\right)$. Since $\theta_{2}$ is coarser than $\theta_{1}$, that means $\theta_{2}\left(\lambda_{A}\right) \subseteq \theta_{1}\left(\lambda_{A}\right)$ for all $\lambda_{A} \in \mathcal{F}_{S S}(X, K)$. This implies, $x_{t}^{h} \widetilde{\notin} \theta_{2}\left(y_{s}^{h^{\prime}}\right)$ and $y_{s}^{h^{\prime}} \widetilde{\notin} \theta_{2}\left(x_{t}^{h}\right)$. Hence, $\left(X, \theta_{2}, K\right)$ is $T_{1}$ - ᄃ́ $\mathcal{F}$-scs. 


\section{T $_{2}$-ČECH FUZZY SOFT CLOSURE SPACES}

In this section we define $T_{2}$ - $\check{C} \mathcal{F}$-scs and other types, namely, semi- (respectively, pseudo and Uryshon) $T_{2}-\check{C} \mathcal{F}$-scs, the properties of each type are discussed as in Section 4 . In addition, the relationships between separation axioms that introduced in the current section and in the previous section are obtained.

Definition 21. A $\check{C} \mathcal{F}$-scs, $(X, \theta, K)$ is said to be $\mathrm{T}_{2}$ - $\check{C} \mathcal{F}$-scs, if for every two distinct fuzzy soft points $x_{t}^{h}$ and $y_{s}^{h^{\prime}}$, there exist disjoint open-fss's $\lambda_{A}$ and $\mu_{B}$ such that $x_{t}^{h} \widetilde{\in} \lambda_{A}$ and $y_{s}^{h^{\prime}} \widetilde{\in} \mu_{B}$.

Example 10. Let $X=\{a, b, c\}, K=\{h\}$. Define $\theta: \mathcal{F}_{\mathrm{SS}}(\mathrm{X}, K) \rightarrow \mathcal{F}_{\mathrm{SS}}(\mathrm{X}, K)$ as follows:

$$
\theta\left(\lambda_{A}\right)=\left\{\begin{array}{cl}
\overline{0}_{K} & \text { if } \lambda_{A}=\overline{0}_{K}, \\
\left\{\left(h, a_{t+0.1} \vee b_{t+0.1}\right)\right\} & \text { if } \lambda_{A} \in\left\{a_{t}^{h} ; 0<t<0.9\right\}, \\
\left\{\left(h, a_{1} \vee c_{1}\right)\right\} & \text { if } \lambda_{A} \in\left\{a_{t}^{h} ; 0.9 \leq t \leq 1\right\}, \\
\left\{\left(h, b_{s+0.1}\right)\right\} & \text { if } \lambda_{A} \in\left\{b_{s}^{h} ; 0<s<0.9\right\}, \\
\left\{\left(h, b_{1}\right)\right\} & \text { if } \lambda_{A} \in\left\{b_{s}^{h} ; 0.9 \leq s \leq 1\right\}, \\
\left\{\left(h, c_{r+0.1}\right)\right\} & \text { if } \lambda_{A} \in\left\{c_{r}^{h} ; 0<r<0.9\right\}, \\
\left\{\left(h, c_{1}\right)\right\} & \text { if } \lambda_{A} \in\left\{c_{r}^{h} ; 0.9 \leq r \leq 1\right\}, \\
\theta\left(a_{t}^{h}\right) \cup \theta\left(b_{s}^{h}\right) & \text { if } \lambda_{A} \in\left\{\left(h, a_{t} \vee b_{s}\right) ; t, s \in I_{0}\right\}, \\
\theta\left(a_{t}^{h}\right) \cup \theta\left(c_{r}^{h}\right) & \text { if } \lambda_{A} \in\left\{\left(h, a_{t} \vee c_{r}\right) ; t, r \in I_{0}\right\}, \\
\theta\left(b_{s}^{h}\right) \cup \theta\left(c_{r}^{h}\right) & \text { if } \lambda_{A} \in\left\{\left(h, b_{s} \vee c_{r}\right) ; s, r \in I_{0}\right\}, \\
\theta\left(a_{t}^{h}\right) \cup \theta\left(b_{s}^{h}\right) \cup \theta\left(c_{r}^{h}\right) & \text { if } \lambda_{A} \in\left\{\left(h, a_{t} \vee b_{s} \vee c_{r}\right) ; t, s, r \in I_{0}\right\} .
\end{array}\right.
$$

Then $(X, \theta, K)$ is $\check{C} \mathcal{F}$-scs. and $(X, \theta, K)$ is $T_{2}$ - $\check{C} \mathcal{F}$-scs. To explain that we have three cases for distinct fuzzy soft points as follows:

$\underline{\text { Case }(1) .} a_{t}^{h}, b_{s}^{h}$ are distinct fuzzy soft points, it follows there exist disjoint open-fss's $\lambda_{A}=\left\{\left(h, a_{1}\right)\right\}$, $\mu_{B}=\left\{\left(h, b_{1}\right)\right\}$ such that $a_{t}^{h} \widetilde{\in} \lambda_{A}$ and $b_{s}^{h} \widetilde{\in} \mu_{B}$.

Case (2). $a_{t}^{h}, c_{s}^{h}$ are distinct fuzzy soft points, it follows there exist disjoint open-fss's $\lambda_{A}=\left\{\left(h, a_{1}\right)\right\}$, $\mu_{B}=\left\{\left(h, c_{1}\right)\right\}$ such that $a_{t}^{h} \widetilde{\in} \lambda_{A}$ and $c_{S}^{h} \widetilde{\in} \mu_{B}$.

$\underline{\text { Case (3). }} b_{s}^{h}, c_{s}^{h}$ are distinct fuzzy soft points, it follows there exist disjoint open-fss's $\lambda_{A}=\left\{\left(h, b_{1}\right)\right\}$, $\mu_{B}=\left\{\left(h, c_{1}\right)\right\}$ such that $a_{t}^{h} \widetilde{\in} \lambda_{A}$ and $c_{S}^{h} \widetilde{\in} \mu_{B}$. Therefore, $(X, \theta, K)$ is $T_{2}$ - $\check{C} \mathcal{F}$-scs.

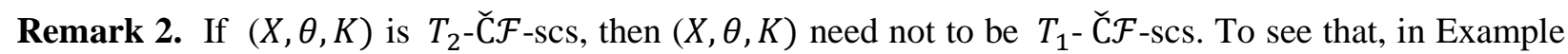
$10,(X, \theta, K)$ is $T_{2}$ - $\check{C} \mathcal{F}$-scs but it is not $(X, \theta, K)$ is $T_{1}$ - $\check{C} \mathcal{F}$-scs. Since there exist $a_{0.5}^{h}$ and $b_{0.5}^{h}$ are distinct fuzzy soft points, and $b_{0.5}^{h} \widetilde{\in} \theta\left(a_{0.5}^{h}\right)$.

In order to study the hereditary property in $\check{C} \mathcal{F}$-scs's, we need first to give the following lemmas.

Lemma 1. Let $(X, \theta, K)$ be a $\check{C} \mathcal{F}$-scs and $\left(V, \theta_{V}, K\right)$ be a $\check{C} \mathcal{F}$-sc subspace of $(X, \theta, K)$. Then for any $\lambda_{A} \in$ $\mathcal{F}_{\text {sS }}(\mathrm{X}, K)$, we have

$$
\left(\overline{1}_{K}-\lambda_{A}\right) \cap \bar{V}_{K}=\bar{V}_{K}-\left(\lambda_{A} \cap \bar{V}_{K}\right) \text {. }
$$

Proof. Let $\lambda_{A} \in \mathcal{F}_{\mathrm{SS}}(\mathrm{X}, K)$. From the definition of $\bar{V}_{K}$, it is clear that for any $h \in K$ and $x \in V, \lambda_{A} \cap \bar{V}_{K}=\lambda_{A}$ . Now, for any $h \in K$ and $x \in V$

$$
\begin{aligned}
{\left[\bar{V}_{K}-\left(\lambda_{A} \cap \bar{V}_{K}\right)\right](h) } & =\left(\bar{V}_{K}-\lambda_{A}\right)(h) \in I^{X} . \text { Now } \\
\left(\bar{V}_{K}-\lambda_{A}\right)(h)(\mathrm{x}) & =\left(\overline{1}_{V}-\lambda_{A}(h)\right)(x) \\
& =\left(\left(\overline{1}_{X}-\lambda_{A}(h)\right) \cap \overline{1}_{V}\right)(x)
\end{aligned}
$$




$$
=\left(\overline{1}_{V}-\lambda_{A}\right) \cap \bar{V}_{K}
$$

Lemma 2. Let $(X, \theta, K)$ be a $\check{C} \mathcal{F}$-scs and let $\left(V, \theta_{V}, K\right)$ be a closed $\check{C} \mathcal{F}$-sc subspace of $(X, \theta, K)$. If $\lambda_{A}$ is an open-fss of $(\mathrm{X}, \theta, K)$ then $\lambda_{A} \cap \bar{V}_{K}$ is also open-fss in $\left(V, \theta_{V}, K\right)$.

Proof. Let $\lambda_{A}$ be an open-fss in $(\mathrm{X}, \theta, K)$. Then $\overline{1}_{K}-\lambda_{A}$ is a closed-fss in $(\mathrm{X}, \theta, K)$. Since $\bar{V}_{K}$ is a closed fuzzy soft set in $(X, \theta, K)$, then $\left(\overline{1}_{K}-\lambda_{A}\right) \cap \bar{V}_{K}$ is a closed-fss in X. That means, $\theta\left(\left(\overline{1}_{K}-\lambda_{A}\right) \cap \bar{V}_{K}\right)=\left(\overline{1}_{K}-\right.$ $\left.\lambda_{A}\right) \cap \bar{V}_{K}$. From Lemma 1, we have $\left(\overline{1}_{K}-\lambda_{A}\right) \cap \bar{V}_{K}=\bar{V}_{K}-\left(\lambda_{A} \cap \bar{V}_{K}\right)$. To complete the prove we must show that $\theta_{V}\left(\bar{V}_{K}-\left(\lambda_{A} \cap \bar{V}_{K}\right)\right)=\bar{V}_{K}-\left(\lambda_{A} \cap \bar{V}_{K}\right)$. Now,

$$
\begin{aligned}
\theta_{V}\left(\bar{V}_{K}-\left(\lambda_{A} \cap \bar{V}_{K}\right)\right) & =\bar{V}_{K} \cap \theta\left(\bar{V}_{K}-\left(\lambda_{A} \cap \bar{V}_{K}\right)\right) & & (\text { By Theorem 2) } \\
& =\bar{V}_{K} \cap \theta\left(\left(\overline{1}_{K}-\lambda_{A}\right) \cap \bar{V}_{K}\right) & & (\text { By Lemma 1) } \\
& =\bar{V}_{K} \cap\left(\left(\overline{1}_{K}-\lambda_{A}\right) \cap \bar{V}_{K}\right) & & \left(\text { since } \theta\left(\left(\overline{1}_{K}-\lambda_{A}\right) \cap \bar{V}_{K}\right)=\left(\overline{1}_{K}-\lambda_{A}\right) \cap \bar{V}_{K}\right) \\
& =\bar{V}_{K} \cap\left(\bar{V}_{K}-\left(\lambda_{A} \cap \bar{V}_{K}\right)\right) & & (\text { By Lemma 1) } \\
& =\bar{V}_{K}-\left(\lambda_{A} \cap \bar{V}_{K}\right) . & &
\end{aligned}
$$

Thus, $\lambda_{A} \cap \bar{V}_{K}$ is an open-fss in $\left(V, \theta_{V}, K\right)$.

Theorem 11. Let $(X, \theta, K)$ be a $T_{2}-\check{C} \mathcal{F}$-scs and let $\left(V, \theta_{V}, K\right)$ be a closed $\check{C} \mathcal{F}$-sc subspace of $(X, \theta, K)$. Then $\left(V, \theta_{V}, K\right)$ is a $T_{2}$-Č $\mathcal{F}$-sc subspace of $(X, \theta, K)$.

Proof. Let $x_{t}^{h}$ and $y_{s}^{h^{\prime}}$ be any two distinct fuzzy soft points in $\left(V, \theta_{V}, K\right)$. Then $x_{t}^{h}$ and $y_{s}^{h^{\prime}}$ are distinct fuzzy soft point in $(X, \theta, K)$. Since $(X, \theta, K)$ is a $T_{2}$ - $\breve{C} \mathcal{F}$-scs, there exist two disjoint open-fss's $\lambda_{A}$ and $\mu_{B}$ such that $x_{t}^{h} \widetilde{\in} \lambda_{A}$ and $y_{s}^{h^{\prime}} \widetilde{\in} \mu_{B}$. Consequently, $x_{t}^{h} \widetilde{\in} \lambda_{A} \cap \bar{V}_{K}, y_{s}^{h^{\prime}} \widetilde{\in} \mu_{B} \cap \bar{V}_{K}$ and $\left(\lambda_{A} \cap \bar{V}_{K}\right) \cap\left(\mu_{B} \cap \bar{V}_{K}\right)=\overline{0}_{K}$. By Lemma $2, \lambda_{A} \cap \bar{V}_{K}$ and $\mu_{B} \cap \bar{V}_{K}$ are open-fss's in $\left(V, \theta_{V}, K\right)$. Hence $\left(V, \theta_{V}, K\right)$ is a $T_{2}$-Č $\mathcal{F}$-sc subspace of $(X, \theta, K)$.

Definition 22. An associative fsts $\left(X, \tau_{\theta}, K\right)$ of $(X, \theta, K)$ is said to be $\mathrm{T}_{2}$-fsts, if for every two distinct fuzzy soft points $x_{t}^{h}$ and $y_{s}^{h^{\prime}}$, there exist an open-fss's $\lambda_{A}$ and $\mu_{B}$ in $\left(X, \tau_{\theta}, K\right)$ such that $x_{t}^{h} \widetilde{\in} \lambda_{A}, y_{s}^{h^{\prime}} \widetilde{\in} \mu_{B}$ and $\lambda_{A} \cap \mu_{B}=\overline{0}_{K}$.

Theorem 12. An associative fsts $\left(X, \tau_{\theta}, K\right)$ is $T_{2}$-fsts of $(X, \theta, K)$ if and only if $(X, \theta, K)$ is $T_{2}$-Č $\mathcal{F}$-scs.

Proof. Suppose $\left(X, \tau_{\theta}, K\right)$ is $T_{2}$-fsts and let $x_{t}^{h}$ and $y_{s}^{h^{\prime}}$ be two distinct fuzzy soft points in $X$. Since $\left(X, \tau_{\theta}, K\right)$ is $T_{2}$-fsts, there exist $\lambda_{A}$ and $\mu_{B}$ open-fss's in $\left(X, \tau_{\theta}, K\right)$ such that $x_{t}^{h} \widetilde{\in} \lambda_{A}, y_{s}^{h^{\prime}} \widetilde{\in} \mu_{B}$ and $\lambda_{A} \cap \mu_{B}=\overline{0}_{K}$. Since $\lambda_{A}$ and $\mu_{B}$ are open-fss's in $\left(X, \tau_{\theta}, K\right)$, then $\tau_{\theta}$-int $\left(\lambda_{A}\right)=\lambda_{A}$ and $\tau_{\theta}$-int $\left(\mu_{B}\right)=\mu_{B}$. From Theorem 4, we get $\operatorname{Int}\left(\lambda_{A}\right)=\lambda_{A}$ and $\operatorname{Int}\left(\mu_{B}\right)=\mu_{B}$. Thus, $\lambda_{A}$ and $\mu_{B}$ are open-fss's in $(X, \theta, K)$ such that $x_{t}^{h} \widetilde{\in} \lambda_{A}$ and $y_{s}^{h^{\prime}} \widetilde{\in} \mu_{B}$ and $\lambda_{A} \cap \mu_{B}=\overline{0}_{K}$. Hence, $(X, \theta, K)$ is $T_{2}$ - $\breve{C} \mathcal{F}$-scs.

Conversely, similar to first direction.

Lemma 3. Let $\left(X, \theta_{1}, K\right),\left(X, \theta_{2}, K\right)$ be $\check{C} \mathcal{F}$-scs's. For any $\lambda_{A} \in \mathcal{F}_{S S}(X, K)$, if $\theta_{1}\left(\lambda_{A}\right) \subseteq \theta_{2}\left(\lambda_{A}\right)$, then $\operatorname{Int}_{2}\left(\lambda_{A}\right) \subseteq \operatorname{Int}_{1}\left(\lambda_{A}\right)$.

Proof. Let $\lambda_{A} \in \mathcal{F}_{\text {sS }}(\mathrm{X}, K)$. From hypothesis, $\theta_{1}\left(\overline{1}_{K}-\lambda_{A}\right) \subseteq \theta_{2}\left(\overline{1}_{K}-\lambda_{A}\right)$, implies $\overline{1}_{K}-\theta_{2}\left(\overline{1}_{K}-\lambda_{A}\right) \subseteq$ $\left.\overline{1}_{K}-\theta_{1}\left(\overline{1}_{K}-\lambda_{A}\right)\right)$. Therefore, $\operatorname{Int}_{2}\left(\lambda_{A}\right) \subseteq \operatorname{Int}_{1}\left(\lambda_{A}\right)$.

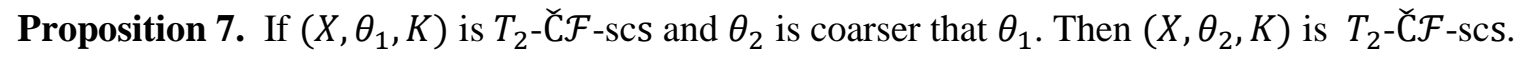

Proof. Let $x_{t}^{h}$ and $y_{S}^{h^{\prime}}$ be any two distinct fuzzy soft points in X. Since $\left(X, \theta_{1}, K\right)$ is a $T_{2}$-Č $\mathcal{F}$-scs, then there exist two disjoint open-fss's $\lambda_{A}$ and $\mu_{B}$ such that $x_{t}^{h} \widetilde{\in} \lambda_{A}$ and $y_{s}^{h^{\prime}} \widetilde{\in} \mu_{B}$. That is mean $\operatorname{Int}_{1}\left(\lambda_{A}\right)=\lambda_{A}$ and $\operatorname{Int}_{1}\left(\mu_{B}\right)=\mu_{B}$. Since $\theta_{2}$ is coarser than $\theta_{1}$, this yields by Lemma $3, \lambda_{A} \subseteq \operatorname{Int}_{1}\left(\lambda_{A}\right) \subseteq \operatorname{Int}_{2}\left(\lambda_{A}\right) \subseteq \lambda_{A}$ and $\mu_{B} \subseteq \operatorname{Int}_{1}\left(\mu_{B}\right) \subseteq \operatorname{Int}_{2}\left(\mu_{B}\right) \subseteq \mu_{B}$. Therefore, there exist two disjoint open-fss's $\lambda_{A}$ and $\mu_{B}$ in $\left(X, \theta_{2}, K\right)$ such that $x_{t}^{h} \widetilde{\in} \lambda_{A}$ and $y_{s}^{h^{\prime}} \widetilde{\in} \mu_{B}$. Hence, $\left(X, \theta_{2}, K\right)$ is $T_{2}$ - $\check{C} \mathcal{F}$-scs. 
Definition 23. A $\check{C} \mathcal{F}$-scs $(X, \theta, K)$ be a is said to be semi $\mathrm{T}_{2}$-Č $\mathcal{F}$-scs, if for every two distinct fuzzy soft points $x_{t}^{h}$ and $y_{s}^{h^{\prime}}$, either there exists an open-fss $\lambda_{A}$ such that $x_{t}^{h} \widetilde{\in} \lambda_{A}$ and $y_{s}^{h^{\prime}} \widetilde{\notin} \theta\left(\lambda_{A}\right)$ or there exists an open-fss $\mu_{B}$ such that $y_{s}^{h^{\prime}} \widetilde{\in} \mu_{B}$ and $x_{t}^{h} \widetilde{\notin} \theta\left(\mu_{B}\right)$.

Example 11. Let $X=\{a, b\}, \mathrm{K}=\{h\}$. Define $\theta: \mathcal{F}_{\mathrm{sS}}(\mathrm{X}, K) \rightarrow \mathcal{F}_{\mathrm{sS}}(\mathrm{X}, K)$ as follows:

$$
\theta\left(\lambda_{A}\right)=\left\{\begin{array}{cl}
\overline{0}_{K} & \text { if } \lambda_{A}=\overline{0}_{K}, \\
b_{1}^{h} & \text { if } \lambda_{A} \subseteq b_{1}^{h}, \\
a_{t+0.1}^{h} & \text { if } \lambda_{A} \in\left\{a_{t}^{h}, 0<t<0.9\right\}, \\
a_{1}^{h} & \text { if } \lambda_{A} \in\left\{a_{t}^{h}, 0 \leq t \leq 1\right\}, \\
\theta\left(a_{t}^{h}\right) \cup \theta\left(b_{s}^{h}\right) & \text { if } \lambda_{A} \in\left\{\left(h, a_{t} \vee b_{s}\right) ; t, s \in I_{0}\right\} .
\end{array}\right.
$$

$(X, \theta, K)$ is semi $T_{2}$-Č $\mathcal{F}$-scs. Since $a_{t}^{h}, b_{s}^{h}$ are distinct fuzzy soft points, there exists an open fuzzy soft set $\lambda_{A}=a_{1}^{h}$ such that $a_{t}^{h} \widetilde{\in} \lambda_{A}$ and $b_{t}^{h} \widetilde{\notin} \theta\left(\lambda_{A}\right)=a_{t}^{h}$.

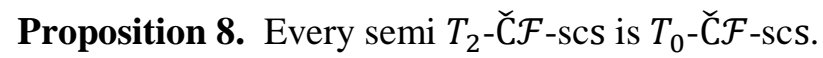

Proof. Let $x_{t}^{h}$ and $y_{s}^{h^{\prime}}$ be any two distinct fuzzy soft points in $(X, \theta, K)$. From $(X, \theta, K)$ is semi $T_{2}$ - $\check{C} \mathcal{F}$-scs, there exists an open-fss $\lambda_{A}$ such that $x_{t}^{h} \widetilde{\in} \lambda_{A}$ and $y_{s}^{h^{\prime}} \widetilde{\notin} \theta\left(\lambda_{A}\right)$ or there exists an open-fss $\mu_{B}$ such that $y_{s}^{h^{\prime}} \widetilde{\in} \mu_{B}$ and $x_{t}^{h} \widetilde{\notin} \theta\left(\mu_{B}\right)$. This implies $x_{t}^{h} \widetilde{\in} \theta\left(x_{t}^{h}\right) \subseteq \theta\left(\lambda_{A}\right)$ and $y_{s}^{h^{\prime}} \widetilde{\notin} \theta\left(x_{t}^{h}\right)$ or $y_{s}^{h^{\prime}} \widetilde{\in} \theta\left(y_{s}^{h^{\prime}}\right) \subseteq$ $\theta\left(\mu_{B}\right)$ and $x_{t}^{h} \widetilde{\notin} \theta\left(y_{s}^{h^{\prime}}\right)$. Hence $(X, \theta, K)$ is a $T_{0}$ - $\breve{C} \mathcal{F}$-scs.

The converse of above proposition is not true.

Example 12. Let $X=\{a, b\}, K=\left\{h_{1} h_{2}\right.$, $\}$ and let $\lambda_{A}^{*} \subseteq \mathcal{F}_{\mathrm{SS}}(\mathrm{X}, K)$ such that $\lambda_{A}^{*}=\left\{\left(h_{1}, a_{t_{1}} \vee b_{s_{1}}\right),\left(h_{2}, a_{t_{2}} \vee b_{s_{2}}\right)\right.$; $\left.t_{1}, t_{2}, s_{1}, s_{2} \in I_{0}\right\}$. Define $\theta: \mathcal{F}_{\text {ss }}(\mathrm{X}, K) \rightarrow \mathcal{F}_{\text {ss }}(\mathrm{X}, K)$ as follows:

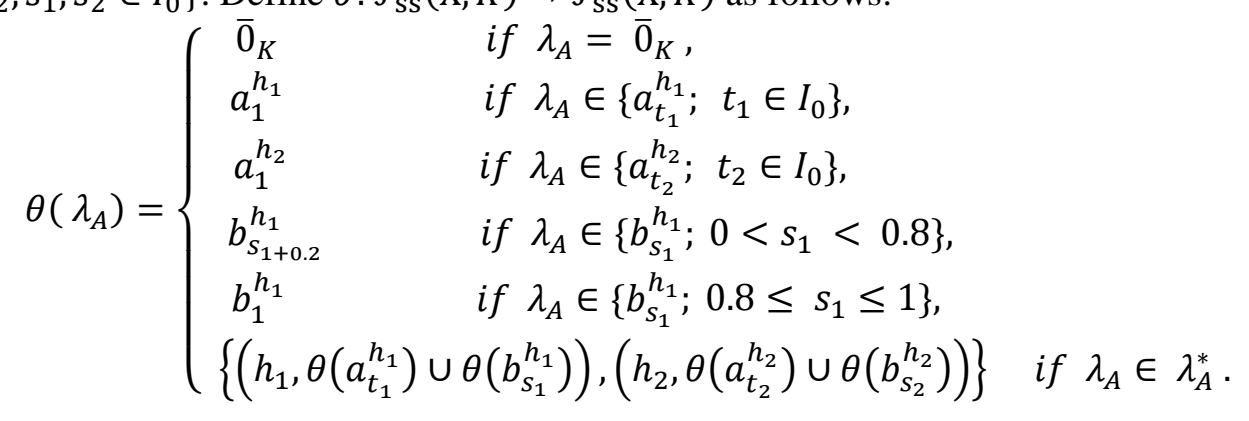

Then $(X, \theta, K)$ is a $T_{0}$ - $\check{C} \mathcal{F}$-scse (see the details in Example 4.4). But $(X, \theta, K)$ is not semi $T_{2}$ - $\check{C} \mathcal{F}$-scs, since there exist $a_{0.5}^{h_{1}}, b_{0.5}^{h_{2}}$ distinct fuzzy soft points such that for any open-fss $\lambda_{A}$, we have $a_{0.5}^{h_{1}} \widetilde{\in} \lambda_{A}$ and $b_{0.5}^{h_{2}} \widetilde{\in} \theta\left(\lambda_{A}\right)$ and for any open-fss $\mu_{B}$, we have $b_{0.5}^{h_{2}} \widetilde{\in} \mu_{B}, a_{0.5}^{h_{1}} \widetilde{\in} \theta\left(\mu_{B}\right)$.

Theorem 13. Let $(X, \theta, K)$ be a semi $T_{2}$-Č $\mathcal{F}$-scs and let $\left(V, \theta_{V}, K\right)$ be a closed $\check{C}$-fsc subspace of $(X, \theta, K)$. Then $\left(V, \theta_{V}, K\right)$ is a semi $T_{2}$-Č $\mathcal{F}$-sc subspace of $(X, \theta, K)$.

Proof. Let $x_{t}^{h}$ and $y_{s}^{h^{\prime}}$ be any two distinct fuzzy soft points in $\left(V, \theta_{V}, K\right)$. Then $x_{t}^{h}$ and $y_{s}^{h^{\prime}}$ are distinct fuzzy soft points in $(X, \theta, K)$. Since $(X, \theta, K)$ is a semi $T_{2}-\check{C} \mathcal{F}$-scs, then either there exists an open-fss $\lambda_{A}$ such that $x_{t}^{h} \widetilde{\in} \lambda_{A}$ and $y_{s}^{h^{\prime}} \widetilde{\notin} \theta\left(\lambda_{A}\right)$ or there exists an open-fss $\mu_{B}$ such that $y_{s}^{h^{\prime}} \widetilde{\in} \mu_{B}$ and $x_{t}^{h} \widetilde{\notin} \theta\left(\mu_{B}\right)$. Now, if $x_{t}^{h} \widetilde{\in} \lambda_{A}$ and $y_{s}^{h^{\prime}} \widetilde{\notin} \theta\left(\lambda_{A}\right)$, then by Lemma $2, x_{t}^{h} \widetilde{\in} \lambda_{A} \cap \bar{V}_{K}$ which is open-fss in $\left(V, \theta_{V}, K\right)$. That is mean we find an open-fss $\lambda_{A} \cap \bar{V}_{K}$ in $\left(V, \theta_{V}, K\right)$ contains $x_{t}^{h}$. To complete the proof, we must show $y_{S}^{h^{\prime}} \widetilde{\notin} \theta_{V}\left(\lambda_{A} \cap \bar{V}_{K}\right)$. It is clear that from the definition of $\theta_{V}$ we have, $\theta_{V}\left(\lambda_{A} \cap \bar{V}_{K}\right)=\bar{V}_{K} \cap \theta\left(\lambda_{A} \cap \bar{V}_{K}\right) \subseteq$ $\bar{V}_{K} \cap \theta\left(\lambda_{A}\right) \cap \theta\left(\bar{V}_{K}\right)=\bar{V}_{K} \cap \theta\left(\lambda_{A}\right)$. And since $y_{s}^{h^{\prime}} \widetilde{\notin} \theta\left(\lambda_{A}\right)$, then we have $y_{s}^{h^{\prime}} \widetilde{\notin} \theta_{V}\left(\lambda_{A} \cap \bar{V}_{K}\right)$. Similarly, if 
there exists an open-fss $\mu_{B}$ such that $y_{s}^{h^{\prime}} \widetilde{\in} \mu_{B}$ and $x_{t}^{h} \widetilde{\notin} \theta\left(\mu_{B}\right)$. Hence, $\left(V, \theta_{V}, K\right)$ is a semi $T_{2}$ - $\check{C} \mathcal{F}$-sc subspace of $(X, \theta, K)$.

Definition 24. An associative fsts $\left(X, \tau_{\theta}, K\right)$ of $(X, \theta, K)$ is said to be semi $T_{2}-f s t s$, if for every distinct fuzzy soft points $x_{t}^{h}$ and $y_{s}^{h^{\prime}}$, either there exists a $\tau_{\theta}$-open-fss $\lambda_{A}$ such that $x_{t}^{h} \widetilde{\in} \lambda_{A}$ and $y_{s}^{h^{\prime}} \widetilde{\notin} \tau_{\theta}$-cl $\left(\lambda_{A}\right)$, or there exists a $\tau_{\theta}$-open-fss $\mu_{B}$ such that $y_{s}^{h^{\prime}} \widetilde{\in} \mu_{B}$ and $x_{t}^{h} \widetilde{\notin} \tau_{\theta}$-cl $\left(\mu_{B}\right)$.

Theorem 14. If $\left(X, \tau_{\theta}, K\right)$ is a semi $T_{2}$-fsts, then $(X, \theta, K)$ is also semi $T_{2}$-Č $\mathcal{F}$-scs.

Proof. Let $x_{t}^{h}$ and $y_{s}^{h^{\prime}}$ be any two distinct fuzzy soft points in $(X, \theta, K)$. From hypothesis, either there exists a $\tau_{\theta}$-open-fss $\lambda_{A}$ such that $x_{t}^{h} \widetilde{\in} \lambda_{A}$ and $y_{s}^{h^{\prime}} \widetilde{\notin} \tau_{\theta}$-cl $\left(\lambda_{A}\right)$, or there exists a $\tau_{\theta}$-open-fss $\mu_{B}$ such that $y_{s}^{h^{\prime}} \widetilde{\in} \mu_{B}$ and $x_{t}^{h} \widetilde{\notin} \tau_{\theta}$-cl $\left(\mu_{B}\right)$. By Theorem 4, we have the following, either there exists an open-fss $\lambda_{A}$ such that $x_{t}^{h} \widetilde{\in} \lambda_{A}$ and $y_{s}^{h^{\prime}} \widetilde{\notin} \theta\left(\lambda_{A}\right)$ or there exists an open-fss $\mu_{B}$ such that $y_{s}^{h^{\prime}} \widetilde{\in} \mu_{B}$ and $x_{t}^{h} \widetilde{\notin} \theta\left(\mu_{B}\right)$. Thus, $(X, \theta, K)$ is a semi $T_{2}$ - $\breve{C} \mathcal{F}$-scs.

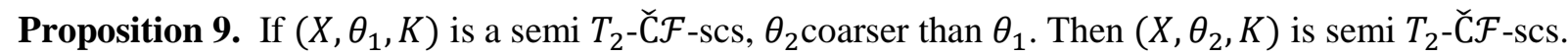

Proof. Let $x_{t}^{h}$ and $y_{s}^{h^{\prime}}$ be any two distinct fuzzy soft points in X. Since $\left(X, \theta_{1}, K\right)$ is semi $T_{2}$ - ᄃ̌ $\mathcal{F}$-scs, then either there exist an open-fss $\lambda_{A}$ such that $x_{t}^{h} \widetilde{\in} \lambda_{A}$ and $y_{s}^{h^{\prime}} \widetilde{\notin} \theta_{1}\left(\lambda_{A}\right)$, or there exist an open-fss $\mu_{B}$ such that $y_{s}^{h^{\prime}} \widetilde{\in} \mu_{B}$ and $x_{t}^{h} \widetilde{\notin} \theta_{1}\left(\mu_{B}\right)$. Suppose, if there exists an open-fss $\lambda_{A}$ in $\left(X, \theta_{1}, H\right)$ that is mean $\operatorname{Int}_{1}\left(\lambda_{A}\right)=\lambda_{A}$. Since $\theta_{2}$ coarser than $\theta_{1}$, then by Lemma 3 , we have $\operatorname{Int}_{1}\left(\lambda_{A}\right) \subseteq \operatorname{Int}_{2}\left(\lambda_{A}\right)$ and that is mean there exists an open-fss $\lambda_{A} \operatorname{in}\left(X, \theta_{2}, K\right)$ such that $x_{t}^{h} \widetilde{\in} \lambda_{A}$. On the other hand since $y_{S}^{h^{\prime}} \widetilde{\notin} \theta_{1}\left(\lambda_{A}\right)$ then $y_{s}^{h^{\prime}} \widetilde{\notin} \theta_{2}\left(\lambda_{A}\right)$. This implies $\left(X, \theta_{2}, K\right)$ is a semi $T_{2}$ - $\check{C} \mathcal{F}$-scs. Similarly, if there exists an open-fss $\mu_{B}$ such that $y_{s}^{h^{\prime}} \widetilde{\in} \mu_{B}$ and $x_{t}^{h} \widetilde{\notin} \theta_{1}\left(\mu_{B}\right)$.

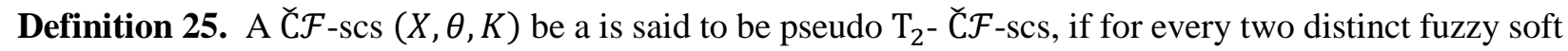
points $x_{t}^{h}$ and $y_{s}^{h^{\prime}}$, there exist open-fss's $\lambda_{A}$ and $\mu_{B}$ such that $x_{t}^{h} \widetilde{\in} \lambda_{A}, y_{s}^{h^{\prime}} \widetilde{\notin} \theta\left(\lambda_{A}\right)$ and $y_{s}^{h^{\prime}} \widetilde{\in} \mu_{B}$, $x_{t}^{h} \widetilde{\notin} \theta\left(\mu_{B}\right)$.

Example 13. Let $X=\{a, b\}, K=\{h\}$. Define $\theta: \mathcal{F}_{\mathrm{sS}}(\mathrm{X}, K) \rightarrow \mathcal{F}_{\mathrm{ss}}(\mathrm{X}, K)$ as follows:

$$
\theta\left(\lambda_{A}\right)=\left\{\begin{array}{cl}
\overline{0}_{K} & \text { if } \lambda_{A}=\overline{0}_{K}, \\
\lambda_{A} & \text { if } \lambda_{A} \in\left\{\left(h, a_{1-t} \vee b_{1}\right) ; 0 \leq t<1\right\}, \\
\lambda_{A} & \text { if } \lambda_{A} \in\left\{\left(h, a_{1} \vee b_{1-s}\right) ; 0 \leq s<1\right\}, \\
\lambda_{A} & \text { if } \lambda_{A} \in\left\{\left(h, a_{t}\right) ; 0<t \leq 1\right\}, \\
\lambda_{A} & \text { if } \lambda_{A} \in\left\{\left(h, b_{s}\right) ; 0<s \leq 1\right\}, \\
\theta\left(a_{t}^{h}\right) \cup \theta\left(b_{S}^{h}\right) & \text { if } \lambda_{A} \in\left\{\left(h, a_{t} \vee b_{s}\right) ; t, s \in I_{0}\right\} .
\end{array}\right.
$$

Then $(X, \theta, K)$ is the discrete $\check{C} \mathcal{F}$-scs. It is clear $\check{C} \mathcal{F}$-scs $(X, \theta, K)$ is pseudo $T_{2}$ - $\check{C} \mathcal{F}$-scs, Since for any $a_{t}^{h}$, $b_{s}^{h}$ are distinct fuzzy soft points there exist an open-fss's $\lambda_{A}=\left\{\left(h, a_{t}\right)\right\}$ and $\mu_{B}=\left\{\left(h, b_{s}\right)\right\}$ such that $a_{t}^{h} \widetilde{\in} \lambda_{A}=\left\{\left(h, a_{t}\right)\right\}, b_{s}^{h} \widetilde{\notin} \theta\left(\lambda_{A}\right)=\lambda_{A}$ and $b_{s}^{h} \widetilde{\in} \mu_{B}=\left\{\left(h, b_{s}\right)\right\}, a_{t}^{h} \widetilde{\notin} \theta\left(\mu_{B}\right)=\mu_{B}$.

Proposition 10. Every pseudo $T_{2}-\check{C} \mathcal{F}$-scs is semi $T_{2}$ - $\check{C} \mathcal{F}$-scs.

Proof. Follows directly from the definition of pseudo $\mathrm{T}_{2^{-}} \check{C} \mathcal{F}$-scs.

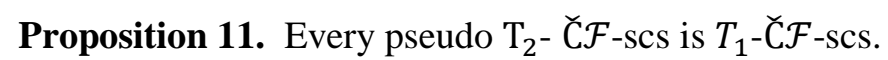

Proof. Let $x_{t}^{h}$ and $y_{S}^{h^{\prime}}$ be any two distinct fuzzy soft points in X. From hypothesis $(X, \theta, K)$ is pseudo $T_{2^{-}}$ $\check{C} \mathcal{F}$-scs, then there exist open-fss's $\lambda_{A}$ and $\mu_{B}$ such that $x_{t}^{h} \widetilde{\in} \lambda_{A}, y_{s}^{h^{\prime}} \widetilde{\notin} \theta\left(\lambda_{A}\right)$ and $y_{s}^{h^{\prime}} \widetilde{\in} \mu_{B}, x_{t}^{h} \widetilde{\notin} \theta\left(\mu_{B}\right)$. 
Since $x_{t}^{h} \widetilde{\in} \lambda_{A}$ and $y_{s}^{h^{\prime}} \widetilde{\in} \mu_{B}$, then it follows $x_{t}^{h} \widetilde{\in} \theta\left(x_{t}^{h}\right) \subseteq \theta\left(\lambda_{A}\right)$ and $y_{s}^{h^{\prime}} \widetilde{\in} \theta\left(y_{s}^{h^{\prime}}\right) \subseteq \theta\left(\mu_{B}\right)$. And since $y_{s}^{h^{\prime}} \widetilde{\notin} \theta\left(\lambda_{A}\right)$ and $x_{t}^{h} \widetilde{\notin} \theta\left(\mu_{B}\right)$, then $y_{s}^{h^{\prime}} \widetilde{\notin} \theta\left(x_{t}^{h}\right)$ and $x_{t}^{h} \widetilde{\notin} \theta\left(y_{s}^{h^{\prime}}\right)$. Hence $(X, \theta, K)$ is a $T_{1}$ - $\breve{C} \mathcal{F}$-scs.

The next example shows that converse of above proposition is not true.

Example 14. Let Let $X=\{a, b\}, \mathrm{K}=\left\{h_{1}, h_{2}\right\}$ and let $\lambda_{A}^{*} \subseteq \mathcal{F}_{\mathrm{sS}}(\mathrm{X}, K)$ such that $\lambda_{A}^{*}=\left\{\left(h_{1}, a_{t_{1}} \vee b_{s_{1}}\right),\left(h_{2}, a_{t_{2}} \vee\right.\right.$ $\left.\left.b_{s_{2}}\right) ; t_{1}, t_{2}, s_{1}, s_{2} \in I_{0}\right\}$. Define $\theta: \mathcal{F}_{\text {ss }}(\mathrm{X}, K) \rightarrow \mathcal{F}_{\text {ss }}(\mathrm{X}, K)$ as follows:

$$
\theta\left(\lambda_{A}\right)=\left\{\begin{array}{cl}
\overline{0}_{K} & \text { if } \lambda_{A}=\overline{0}_{K}, \\
a_{1}^{h_{1}} & \text { if } \lambda_{A} \subseteq a_{1}^{h_{1}}, \\
a_{1}^{h_{2}} & \text { if } \lambda_{A} \subseteq a_{1}^{h_{2},} \\
b_{1}^{h_{1}} & \text { if } \lambda_{A} \subseteq b_{1}^{h_{1}}, \\
b_{0.9}^{h_{2}} & \text { if } \lambda_{A} \in\left\{b_{s}^{h_{2}} ; 0<s<0.9\right\}, \\
b_{1}^{h_{2}} & \text { if } \lambda_{A} \in\left\{b_{s}^{h_{2}} ; 0.9 \leq s \leq 1\right\}, \\
\left\{\left(h_{1}, \theta\left(a_{t_{1}}^{h_{1}}\right) \cup \theta\left(b_{s_{1}}^{h_{1}}\right)\right),\left(h_{2}, \theta\left(a_{t_{2}}^{h_{2}}\right) \cup \theta\left(b_{s_{2}}^{h_{2}}\right)\right)\right\} \quad \text { if } \lambda_{A} \in \lambda_{A}^{*} .
\end{array}\right.
$$

Then $(X, \theta, K)$ is $T_{1}$-Č $\mathcal{F}$-scs. But $(X, \theta, K)$ is not pseudo $T_{2}$ - $\check{C} \mathcal{F}$-scs. To show that consider $a_{0.5}^{h_{1}}$ and $b_{0,7}^{h_{2}}$ are distinct fuzzy soft points. The open-fss's $\lambda_{A}$ such that $a_{0.5}^{h_{1}} \widetilde{\in} \lambda_{A}$ are:

1. $\lambda_{A}=\overline{1}_{H}$, implies $b_{0.7}^{h_{2}} \widetilde{\in} \theta\left(\overline{1}_{H}\right)$.

2. $\lambda_{A}=\left\{\left(h_{1}, a_{1} \vee b_{1}\right),\left(h_{1}, b_{1}\right)\right\}$, implies $b_{0.7}^{h_{2}} \widetilde{\in} \theta\left(\lambda_{A}\right)$.

3. $\lambda_{A}=\left\{\left(h_{1}, a_{1}\right),\left(h_{2}, a_{1} \vee b_{1}\right)\right\}$, implies $b_{0.7}^{h_{2}} \widetilde{\in} \theta\left(\lambda_{A}\right)$.

4. $\lambda_{A}=\left\{\left(h_{1}, a_{1} \vee b_{1}\right),\left(h_{2}, a_{1}\right)\right\}$, implies $b_{0.7}^{h_{2}} \widetilde{\in} \theta\left(\lambda_{A}\right)$.

Hence, for all open-fss $\lambda_{A}$ such that $a_{0.5}^{h_{1}} \widetilde{\in} \lambda_{A}$, we have $b_{0,7}^{h_{2}} \widetilde{\in} \theta\left(\lambda_{A}\right)$. Thus, $(X, \theta, K)$ is not pseudo $T_{2}$ - $\breve{C} \mathcal{F}$ scs.

Theorem 15. Let $(X, \theta, K)$ be a pseudo $T_{2}$ - $\check{C} \mathcal{F}$-scs and let $\left(V, \theta_{V}, K\right)$ be a closed $\check{C}$-fsc subspace of

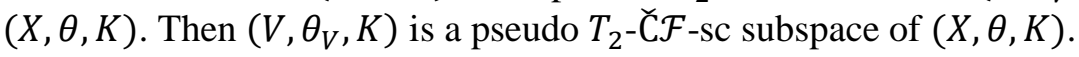

Proof: Similar of Theorem 13.

Definition 26. An associative fsts $\left(X, \tau_{\theta}, K\right)$ of $(X, \theta, K)$ is said to be a pseudo $\mathrm{T}_{2}$-fsts, if for every distinct fuzzy soft points $x_{t}^{h}$ and $y_{s}^{h^{\prime}}$ there exist $\tau_{\theta}$-open-fss's $\lambda_{A}$ and $\mu_{B}$ such that $x_{t}^{h} \widetilde{\in} \lambda_{A}, y_{s}^{h^{\prime}} \widetilde{\notin} \tau_{\theta}$-cl( $\left.\lambda_{A}\right)$ and $y_{s}^{h^{\prime}} \widetilde{\in} \mu_{B}$ and $x_{t}^{h} \widetilde{\notin} \tau_{\theta^{-}} c l\left(\mu_{B}\right)$.

Theorem 16. If $\left(X, \tau_{\theta}, K\right)$ is a pseudo $T_{2}$-fsts, then $(X, \theta, K)$ is also pseudo $T_{2}-\check{C} \mathcal{F}$-scs.

Proof. Similar of Theorem 14.

Proposition 12. If $\left(X, \theta_{1}, K\right)$ be a pseudo $T_{2}$ - $\check{\mathcal{C}} \mathcal{F}$-scs, $\theta_{2}$ coarser than $\theta_{1}$, then $\left(X, \theta_{2}, K\right)$ is pseudo $T_{2}$ - $\check{C} \mathcal{F}$ scs.

Proof. Similar of proof Proposition 9.

Definition 27. A $\check{C} \mathcal{F}$-scs $(X, \theta, K)$ be a is said to be Uryshon $\mathrm{T}_{2}$ - $\check{C} \mathcal{F}$-scs, if for every two distinct fuzzy soft points $x_{t}^{h}$ and $y_{s}^{h^{\prime}}$, there exist open-fss's $\lambda_{A}$ and $\mu_{B}$ such that $x_{t}^{h} \widetilde{\in} \lambda_{A}, y_{s}^{h^{\prime}} \widetilde{\in} \mu_{B}$ and $\theta\left(\lambda_{A}\right)$ $\cap \theta\left(\mu_{B}\right)=\overline{0}_{K}$.

Example 15. Let $X=\{a, b\}, K=\{h\}$. Define $\theta: \mathcal{F}_{\mathrm{SS}}(\mathrm{X}, K) \rightarrow \mathcal{F}_{\mathrm{SS}}(\mathrm{X}, K)$ as follows: 


$$
\theta\left(\lambda_{A}\right)= \begin{cases}\overline{0}_{K} & \text { if } \lambda_{A}=\overline{0}_{K}, \\ a_{1}^{h} & \text { if } \lambda_{A} \subseteq a_{1}^{h}, \\ b_{1}^{h} & \text { if } \lambda_{A} \subseteq b_{1}^{h}, \\ \overline{1}_{K} & \text { other wise. }\end{cases}
$$

Then $(X, \theta, K)$ is a Uryshon $T_{2}$ - $\breve{C} \mathcal{F}$-scs. Since Consider $a_{t}^{h}$ and $b_{s}^{h}$ are distinct fuzzy soft points, there exist open-fss's $\lambda_{A}=a_{1}^{h}$ and $\mu_{B}=b_{1}^{h}$ such that $a_{t}^{h} \widetilde{\in} \lambda_{A}, b_{s}^{h} \widetilde{\in} \lambda_{A}$ and $\theta\left(\lambda_{A}\right) \cap \theta\left(\mu_{B}\right)=a_{1}^{h} \cap b_{1}^{h}=\overline{0}_{K}$.

Proposition 13. Every Uryshon $T_{2}$-Č $\mathcal{F}$-scs is pesudo $T_{2}$ - $\check{C} \mathcal{F}$-scs.

Proof. Let $x_{t}^{h}$ and $y_{S}^{h^{\prime}}$ be any two-distinct fuzzy soft points in $(X, \theta, H)$. Since $(X, \theta, H)$ is Uryshon $T_{2^{-}}$ $\check{C} \mathcal{F}$-scs, there exist $\lambda_{A}$ and $\mu_{B}$ open fuzzy soft sets such that $x_{t}^{h} \widetilde{\in} \lambda_{A}, y_{s}^{h^{\prime}} \widetilde{\in} \mu_{B}$ and $\theta\left(\lambda_{A}\right) \cap \theta\left(\mu_{B}\right)=\overline{0}_{K}$. This implies $x_{t}^{h} \widetilde{\notin} \theta\left(\mu_{B}\right)$ and $y_{s}^{h^{\prime}} \widetilde{\notin} \theta\left(\lambda_{A}\right)$. Therefore, $(X, \theta, K)$ is pesudo $T_{2}-\check{C} \mathcal{F}$-scs.

Proposition 14. Every Uryshon $T_{2}$ - $\check{C} \mathcal{F}$-scs is $T_{2}$ - $\check{C} \mathcal{F}$-scs.

Proof. The proof follows immediately from the definition of Uryshon $T_{2}-\check{C} \mathcal{F}$-scs and the property $\lambda_{A} \subseteq$ $\theta\left(\lambda_{A}\right)$ for any fuzzy soft set $\lambda_{A}$.

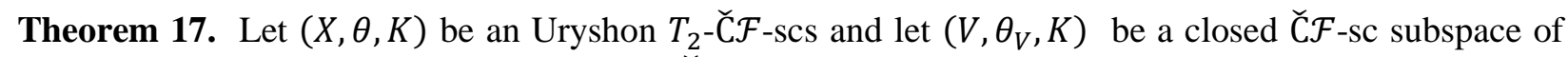

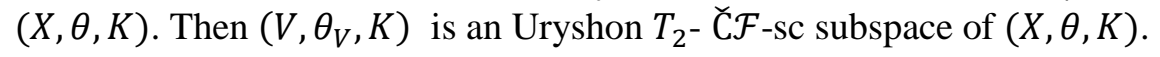

Proof. Let $x_{t}^{h}$ and $y_{s}^{h^{\prime}}$ be any two-distinct fuzzy soft points in $\left(V, \theta_{V}, K\right)$. Then $x_{t}^{h}$ and $y_{s}^{h^{\prime}}$ are distinct fuzzy soft points in $(X, \theta, K)$. Since $(X, \theta, K)$ is a Uryshon $T_{2}-\check{C} \mathcal{F}$-scs, it follows there exist open-fss's $\lambda_{A}$ and $\mu_{B}$ such that $x_{t}^{h} \widetilde{\in} \lambda_{A}, y_{s}^{h^{\prime}} \widetilde{\in} \mu_{B}$ and $\theta\left(\lambda_{A}\right) \cap \theta\left(\mu_{B}\right)=\overline{0}_{K}$. By Lemma 2, $\lambda_{A} \cap \bar{V}_{K}$ and $\mu_{B} \cap \bar{V}_{K}$ are open fuzzy soft sets in $\bar{V}_{K}$ such that $x_{t}^{h} \widetilde{\in} \lambda_{A} \cap \bar{V}_{K}, y_{s}^{h^{\prime}} \widetilde{\in} \mu_{B} \cap \bar{V}_{K}$. Next, we must show that $\theta_{V}\left(\lambda_{A} \cap \bar{V}_{K}\right) \cap$ $\theta_{V}\left(\mu_{B} \cap \bar{V}_{K}\right)=\overline{0}_{K}$. Now, from the definition of $\theta_{V}$ we get,

$$
\begin{aligned}
\theta_{V}\left(\lambda_{A} \cap \bar{V}_{K}\right) \cap \theta_{V}\left(\mu_{B} \cap \bar{V}_{K}\right) & =\left[\bar{V}_{K} \cap \theta\left(\lambda_{A} \cap \bar{V}_{K}\right)\right] \cap\left[\bar{V}_{K} \cap \theta\left(\mu_{B} \cap \bar{V}_{K}\right)\right] \\
& =\left[\theta\left(\lambda_{A}\right) \cap \theta\left(\mu_{B}\right)\right] \cap \bar{V}_{K} \\
& =\overline{0}_{K} \cap \bar{V}_{K} \\
& =\overline{0}_{K} .
\end{aligned}
$$

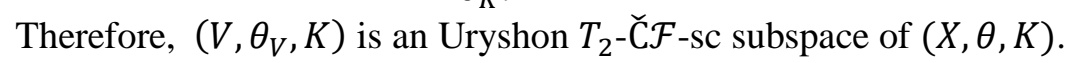

Definition 28. An associative fsts $\left(X, \tau_{\theta}, K\right)$ of $(X, \theta, K)$ is said to be a Uryshon $\mathrm{T}_{2}$-fst, if for every distinct fuzzy soft points $x_{t}^{h}$ and $y_{s}^{h^{\prime}}$, there exist $\tau_{\theta}$-open fuzzy soft sets $\lambda_{A}$ and $\mu_{B}$ such that $x_{t}^{h} \widetilde{\in} \lambda_{A}, y_{s}^{h^{\prime}} \widetilde{\in} \mu_{B}$ and $\tau_{\theta}-c l\left(\lambda_{A}\right) \cap \tau_{\theta}-c l\left(\mu_{B}\right)=\overline{0}_{K}$.

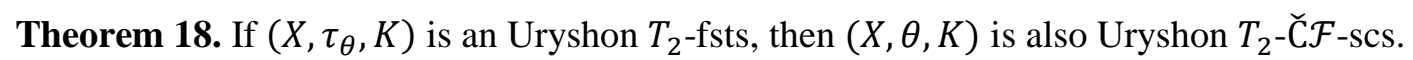

Proof: Let $x_{t}^{h}$ and $y_{s}^{h^{\prime}}$ be any two-distinct fuzzy soft points in $X$. Since $\left(X, \tau_{\theta}, K\right)$ is an Uryshon $T_{2}$-fsts, then there exist $\tau_{\theta}$-open-fss's $\lambda_{A}$ and $\mu_{B}$ such that $x_{t}^{h} \widetilde{\in} \lambda_{A}, y_{s}^{h^{\prime}} \widetilde{\in} \mu_{B}$ and $\tau_{\theta}-\operatorname{cl}\left(\lambda_{A}\right) \cap \tau_{\theta}-\operatorname{cl}\left(\mu_{B}\right)=\overline{0}_{K}$. By Theorem 4, we obtain $\lambda_{A}$ and $\mu_{B}$ are open fuzzy soft sets in $(X, \theta, K)$ such that $x_{t}^{h} \widetilde{\epsilon} \lambda_{A}$ and $y_{s}^{h^{\prime}} \widetilde{\epsilon} \mu_{B}$ and $\theta\left(\lambda_{A}\right) \cap \theta\left(\mu_{B}\right)=\overline{0}_{K}$. Hence, $(X, \theta, K)$ is an Uryshon $T_{2}$-Č $\mathcal{F}$-scs.

Proposition 15. If $\left(X, \theta_{1}, K\right)$ be a Uryshon $T_{2}$ - $\breve{C} \mathcal{F}$-scs, $\theta_{2}$ coarser than $\theta_{1}$, then $\left(X, \theta_{2}, K\right)$ is Uryshon $T_{2}$ $\check{\mathrm{C}} \mathcal{F}$-scs.

Proof. Similar of proof Proposition 9. 
Remark 3. The relationships between the above types of separation axioms in ČF-scs's as shown in the following diagram.

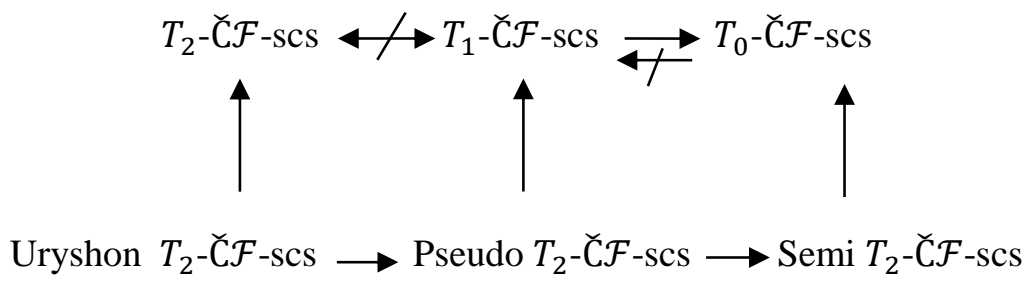

\section{CONFLICTS OF INTEREST}

No conflict of interest was declared by the authors.

\section{REFERENCES}

[1] Zadeh, L. A., "Fuzzy sets", Information and Control, 8: 338-353, (1965).

[2] Molodtsov, D. A., "Soft set theory first results", Computers and Mathematics with Applications, 37: 19-31, (1999).

[3] Maji, P. K., Biswas, R. and Roy, A. R., "Fuzzy soft sets", Journal of Fuzzy Mathematics, 9(3): 589$602,(2001)$.

[4] Tanay, B. and Kandemir, M. B., "Topological structures of fuzzy soft sets", Computers and Mathematics with Applications, 61: 412-418, (2011).

[5] $\breve{C}$ ech, E., Topological spaces, Inter Science Publishers, John Wiley and Sons, New York, (1966).

[6] Mashhour, A. S. and Ghanim, M. H., "Fuzzy closure spaces", Journal of Mathematical Analysis and Applications, 106: 154-170, (1985).

[7] Gowri, R. and Jegadeesan, G., "On soft $\breve{C}$ ech closure spaces", International Journal of Mathematics Trends and Technology, 9(2): 122-127, (2014).

[8] Krishnaveni, J. and Sekar, C., "Čech Soft Closure Spaces”, International Journal of Mathematical Trends and Technology, 6: 123-135, (2014).

[9] Majeed, R. N.,“Čech fuzzy soft closure spaces”, International Journal of Fuzzy System Applications, 7(2): 62-74, (2018).

[10] Majeed, R. N. and Maibed, L.H., "Some structures of Čech fuzzy soft closure spaces", Journal of Engineering and Applied Sciences, Accepted.

[11] Roy, S. and Samanta, T. K., "A note on fuzzy soft topological spaces”, Annals of Fuzzy Mathematics and Informatics, 3(2): 305-311, (2012).

[12] Varol,B. P. and Aygün, H. "Fuzzy soft topology", Hacettepe Journal of Mathematics and Statistics, 41(2): 407-419, (2012).

[13] Mahanta, J. and Das, P.K., "Results on fuzzy soft topological spaces”, arXiv:1203.0634v, (2012).

[14] Atmaca, S. and Zorlutuna, İ., "On fuzzy soft topological spaces", Annals of Fuzzy Mathematics and Informatics, 5(2): 377-386, (2013). 
[15] Mishra, S. and Srivastava, R., "Hausdorff fuzzy soft topological spaces", Annals of Fuzzy Mathematics and Informatics, 9(2): 247-260, (2015). 